\title{
The variation of flow and turbulence across the sediment-water interface
}

\author{
J. J. Voermans ${ }^{1, \dagger}$, M. Ghisalberti ${ }^{1,2}$ and G. N. Ivey ${ }^{1,3}$ \\ ${ }^{1}$ School of Civil, Environmental and Mining Engineering, University of Western Australia, Crawley, \\ WA 6009, Australia \\ ${ }^{2}$ Department of Infrastructure Engineering, University of Melbourne, Parkville, VIC 3010, Australia \\ ${ }^{3}$ The UWA Oceans Institute, University of Western Australia, Crawley, WA 6009, Australia
}

(Received 8 November 2016; revised 16 May 2017; accepted 18 May 2017;

first published online 6 July 2017)

A basic framework characterising the interaction between aquatic flows and permeable sediment beds is presented here. Through the permeability Reynolds number $\left(R e_{K}=\sqrt{K} u_{*} / \nu\right.$, where $K$ is the sediment permeability, $u_{*}$ is the shear velocity and $v$ is the fluid viscosity), the framework unifies two classical flow typologies, namely impermeable boundary layer flows $\left(R e_{K} \ll 1\right)$ and highly permeable canopy flows $\left(R e_{K} \gg 1\right)$. Within this range, the sediment-water interface (SWI) is identified as a transitional region, with $R e_{K}$ in aquatic systems typically $O(0.001-10)$. As the sediments obstruct conventional measurement techniques, experimental observations of interfacial hydrodynamics remain extremely rare. The use of refractive index matching here allows measurement of the mean and turbulent flow across the SWI and thus direct validation of the proposed framework. This study demonstrates a strong relationship between the structure of the mean and turbulent flow at the SWI and $R e_{K}$. Hydrodynamic characteristics, such as the interfacial turbulent shear stress, velocity, turbulence intensities and turbulence anisotropy tend towards those observed in flows over impermeable boundaries as $R e_{K} \rightarrow 0$ and towards those seen in flows over highly permeable boundaries as $R e_{K} \rightarrow \infty$. A value of $R e_{K} \approx 1-2$ is seen to be an important threshold, above which the turbulent stress starts to dominate the fluid shear stress at the SWI, the penetration depths of turbulence and the mean flow into the sediment bed are comparable and similarity relationships developed for highly permeable boundaries hold. These results are used to provide a new perspective on the development of interfacial transport models at the SWI.

Key words: shear layer turbulence, turbulent boundary layers, turbulent mixing

\section{Introduction}

The vertical transport of mass and momentum in aquatic flows is profoundly impacted by the complex interactions between the turbulent flow and the sediments. The extent to which the sediments influence the chemical and biological composition of the water column and the frictional resistance to the overlying flow is determined

$\dagger$ Email address for correspondence: joey.voermans@ research.uwa.edu.au 
by the rate at which mass and momentum are transferred across the sediment-water interface (SWI). Transport processes at the interface are thus of primary interest in any assessment of water quality, aquatic ecosystem health, flood control and coastal management. For instance, sediments act as both a source and sink for contaminants, such as heavy metals. These contaminants are often able to redissolve in the interstitial fluid and can subsequently return to the water column through interfacial mixing processes (Ciceri et al. 1992; Blasco, Saenz \& Gómez-Parra 2000), exposing the ecosystem to contaminants in the long term. Moreover, the interstitial water in sediment beds is typically characterised by elevated levels of nutrients and depleted levels of dissolved oxygen relative to the water column. Periods of significant oxygen undersupply to the sediments can generate zones of hypoxia, which can affect the ecosystem significantly (Diaz 2001). As oxygen and nutrient concentrations in sediments are important determinants of nutrient transformation and microbial metabolism in aquatic ecosystems, the permeability of sediments is demonstrably linked to the health of downstream ecosystems (Rabalais et al. 2001; Battin et al. 2016).

In addition to the role of sediments in determining aquatic ecosystem health, the dynamics of the interfacial flow determine the transport of momentum to the sediments and hence the flow resistance (i.e. the bed shear stress). Quantification of the bed shear stress is imperative in river management and coastal defence (Horritt $\&$ Bates 2002), as the shear stress governs river water levels and determines rates of sediment resuspension and transport. Although the bed shear stress is generally applied as a temporally and spatially averaged variable, the stress fluctuates in both time and space, with both variations inextricably linked to turbulent structures in the overlying flow (Mignot, Barthelemy \& Hurther 2009; Mathis et al. 2014). It is also suggested that these fluctuations are fundamental to sediment entrainment (Nelson et al. 1995). The nature of the mean flow and turbulence at the SWI remain largely unknown, yet fundamental knowledge of these dynamics is required in order to accurately predict the interfacial transfer of mass and momentum.

Advances in our understanding of the interfacial dynamics are currently hampered by a lack of detailed measurements, because of the obstruction presented by, and the small length scales of, sediment beds (Boulton et al. 1998; Packman, Salehin \& Zaramella 2004). This lack of empirical information on the dynamics of interactions between the flow and sediments is currently bypassed by using simplified conceptual models to predict interfacial transport. Traditional models used to predict mass and momentum transport across the SWI often assume a negligible permeability of the sediments, enabling the use of theoretical concepts from the study of flows over impermeable boundaries. For example, modelling momentum transport at a smooth impermeable boundary has traditionally relied on the concept of a viscous sublayer (within which viscosity damps turbulent fluctuations) adjacent to the boundary. In environmental flows, the thickness of the viscous sublayer is typically smaller than, or at most comparable to, the size of the roughness elements on the bed, such that the viscous sublayer is submerged within the roughness and undulates with the bed topography (due to the no-slip condition) (e.g. Tennekes \& Lumley 1972).

Similarly, the modelling of mass transport across the SWI is historically based on the concept of a thin layer close to the wall, the diffusive boundary layer (DBL), where molecular diffusion dominates mass transport. For a rough boundary, it is argued that the conceptual DBL (like the viscous sublayer) is submerged in the roughness elements and follows the topography of the bed (Jorgensen \& Des Marais 1990). The validity of the DBL model is questionable, however, as measured rates 
of transfer are generally much larger than can be explained by molecular diffusion alone and show strong correlations with turbulent structures in the flow (Lorke et al. 2003; Hondzo et al. 2005; O'Connor \& Harvey 2008). It has also been found that direct exchange of mass, such as dispersion around sediment grains (Güss 1998) and advection by turbulent eddies (Packman et al. 2004), can dominate the interfacial transport. These observations are not compatible with the assumption of sediment impermeability and thus greatly restrict the use of the DBL model in predicting interfacial fluxes.

Relaxing the assumption of impermeability allows the turbulent flow to penetrate the SWI and hence transport processes (beyond molecular diffusion) to directly transfer material. Two modelling approaches are common, either coupling the flow above the interface to the interstitial fluid through interfacial boundary conditions (Beavers \& Joseph 1967) or by assuming a continuous variation of properties (such as the porosity and the eddy viscosity) in the vertical (Ruff \& Gelhar 1972). Both approaches are referred to as 'slip models' as they allow flow penetration into the porous medium but are substantially different as the coupling is done by means of either an interfacial velocity, which assumes laminar flow inside the porous medium, or by an eddy viscosity model, which allows turbulence to penetrate the porous medium. As the interface is a region of transition, local gradients in velocity and porosity are large, which makes the commonly used boundary conditions at the interface sensitive to both the position chosen for the interface (Saffman 1971) and the topography of the sediment bed (Goyeau et al. 2003). This requires detailed observations of the variation of flow properties across the SWI as input for the slip models, information that is currently unavailable.

The practical and physical limitations of both the DBL model and the slip model have led to the development of empirical formulations for the interfacial diffusivity (e.g. O'Connor \& Harvey 2008; Grant, Stewardson \& Marusic 2012). These formulations are based on multiple linear regression analysis of experimental data, but do not shed light on the underlying physical processes. They remain, however, instrumental in identifying relevant parameters in the description of mass and momentum transport. The limitations of empirical formulations and the distinct conceptual differences between the DBL model and the slip model demonstrate the need for a more fundamental understanding of the interfacial dynamics.

The purpose of this study is thus to undertake a series of novel experimental observations in order to provide a framework for characterising the hydrodynamic processes that determine mass and momentum transfer across the SWI. The experimental data will reveal the variation of the mean flow and turbulence across the SWI as a function of a dimensionless permeability. These experimental results will also clarify the limitations of both the DBL model and the slip model by evaluation of the assumptions that underpin them.

\section{A hydrodynamic framework}

\subsection{Spatial averaging}

As flow properties are highly spatially heterogeneous near irregular rough boundaries, spatial averaging is essential to provide reliable estimates of the flow properties. The double-averaging procedure was used here, whereby the Reynolds decomposition ( $\xi=\bar{\xi}+\xi^{\prime}$, where $\xi$ is the flow variable) is accompanied by a spatial decomposition of the time-averaged variable $(\bar{\xi}=\langle\bar{\xi}\rangle+\tilde{\xi}$, where angular brackets denote the horizontal average and $\tilde{\xi}$ the fluctuation in space) (Nikora et al. 2007). Properties are averaged 
over the fluid domain $V_{f}$, giving the intrinsic spatial average $\langle\xi\rangle=1 / V_{f} \int_{V_{f}} \xi \mathrm{d} V$. Substitution of the spatial decomposition into the Navier-Stokes equations leads to additional terms, referred to as form-induced terms, and represent the spatial correlations of time-averaged quantities. The distribution of combined forces per unit area in the horizontal plane is obtained by integrating the simplified two-dimensional spatially averaged Navier-Stokes equations (Nikora et al. 2001, 2004):

$$
\tau_{R}(z)=\mu \frac{\partial \theta\langle\bar{u}\rangle}{\partial z}-\rho \theta\left\langle\overline{\left\langle u^{\prime} w^{\prime}\right.}\right\rangle-\rho \theta\langle\tilde{u} \tilde{w}\rangle+f_{f}+f_{v},
$$

where $u, v$ and $w$ are the velocity components in the directions of $x, y$ and $z, \tau_{R}$ is the total force per unit area in the horizontal plane, $\mu$ the dynamic viscosity and $\rho$ the fluid density. $\theta(z)$ is the porosity function with respect to $z$, which takes on a value of 1 above the sediment bed and reaches a constant value $\theta_{p}$ well within the sediment bed. The terms in (2.1) are the viscous stress $\left(\tau_{v}\right)$, turbulent stress $\left(\tau_{t}\right)$, form-induced stress $\left(\tau_{f}\right)$, form drag per unit area $\left(f_{f}\right)$ and viscous drag per unit area $\left(f_{v}\right)$ respectively. Note that the viscous, turbulent and form-induced stresses represent fluid stresses acting in the fluid domain (the sum defines the total fluid shear stress $\tau$ ), while the viscous and form drag represent forces acting at the surfaces of the sediment grains. The fluid stresses relate to a flux of momentum while drag forces relate to the momentum sink. The relevance of each term depends on $z$, the properties of the flow and the properties of the boundary. $f_{f}$ and $f_{v}$ for instance are both zero above the roughness crests of the sediment bed.

\subsection{Permeability Reynolds number}

In description of either transport across the SWI or the flow properties above the interface, many different velocity and length scales have been employed. Examples include the velocity at the top of the boundary layer $U_{\delta}$, the shear velocity $u_{*}$, the boundary layer thickness $\delta$ defined as $\langle\bar{u}\rangle_{z=\delta}=0.99 U_{\infty}$, the size of the roughness elements $k_{s}$, the square root of the sediment permeability $\sqrt{K}$ and the thickness of the porous medium $H_{s}$. Thus, a variety of Reynolds numbers have been used to characterise the flow: $R e=U_{\delta} \delta / v, R e_{*}=u_{*} \delta / \nu, R e_{k_{s}}=u_{*} k_{s} / v$ and the permeability Reynolds number $R e_{K}=u_{*} \sqrt{K} / v$, where $v$ is the kinematic viscosity. The sediment bed thickness $\left(H_{s}\right)$ becomes relevant only when it restricts the depth of flow penetration (e.g. Prinos, Sofialidis \& Keramaris 2003). When this restriction occurs, the boundary is not considered fully permeable and the flow will be strongly dependent on the dimensionless bed thickness, $H_{s} / \sqrt{K}$. Here, we only consider fully permeable boundaries, defined as a boundary for which the depth of penetration of the mean flow and turbulence are independent of $H_{s}$. Both $R e$ and $R e_{*}$ characterise the overlying flow and do not take into account the physical properties of the SWI. Both $R e_{k_{s}}$ and $R e_{K}$ are dynamically relevant to the flow at the SWI, and both have been used to describe the near-boundary hydrodynamics (e.g. Jiménez 2004; Breugem, Boersma \& Uittenbogaard 2006) and enhanced exchange across the SWI (O'Connor \& Harvey 2008). For granular beds, $\sqrt{K}$ and $k_{s}$ are tightly linked (e.g. Wilson, Huettel \& Klein 2008). In particular, for a bed composed of monodisperse spherical particles, $k_{s} / \sqrt{K} \approx 9$. Moreover, it is argued that as the depth of penetration increases with $\operatorname{Re}_{K}$, the roughness length scale seen by the overlying flow increases as well, thereby inextricably linking $\operatorname{Re}_{K}$ and $R e_{k_{s}}$ (Manes et al. 2011b; Manes, Ridolfi \& Katul 2012). The relationship between $\sqrt{K}$ and $k_{s}$ may be highly variable in other 


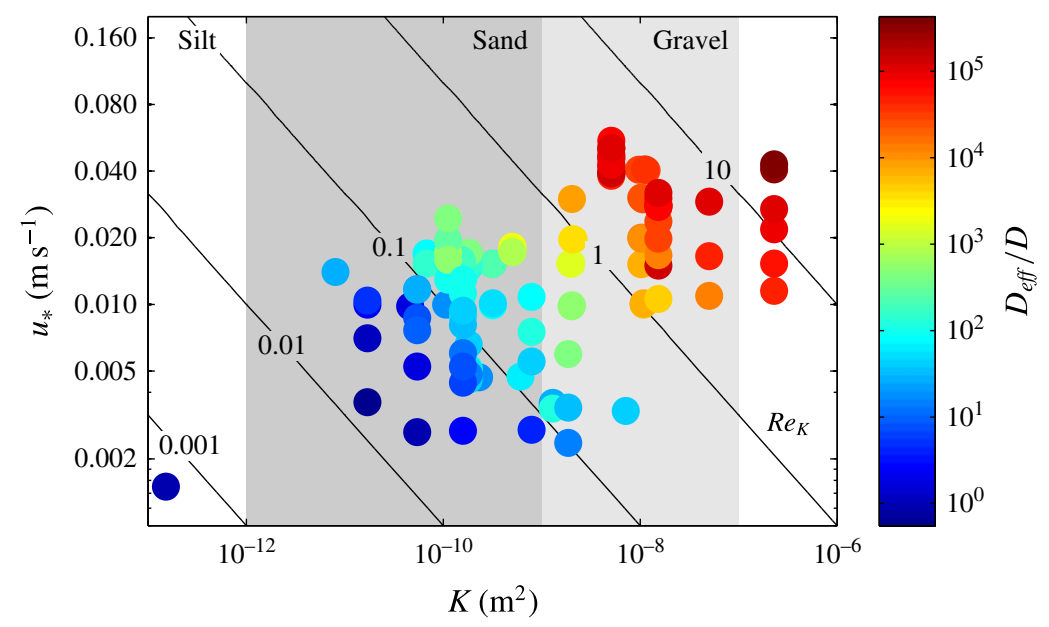

FIgURE 1. The dependence of mass transfer at the SWI on $\operatorname{Re}_{K}$. The colour of the markers represents the ratio of the effective diffusivity at the SWI to the coefficient of molecular diffusion $\left(D_{\text {eff }} / D\right)$; data are taken from Roy et al. (2004), Grant et al. (2012) and Chandler et al. (2016). Diagonal lines of constant $\operatorname{Re}_{K}$ are shown, assuming $v=$ $1 \times 10^{-6} \mathrm{~m}^{2} \mathrm{~s}^{-1}$. Sediment classifications (from Bear 1972) are given in grey. A strong correlation exists between interfacial mass transport and $R e_{K}$; for $R e_{K} \geqslant O(0.1)$, molecular diffusion becomes negligible in interfacial transport.

porous media, thus restricting the application of this study to granular beds. As the importance of $R e_{K}$ in governing interfacial fluxes of momentum (e.g. Ruff \& Gelhar (1972), Breugem et al. (2006) and implicitly in Manes et al. (2012)) and mass (Grant et al. 2012) has been clearly identified, and the influence of the roughness elements is implicitly taken into account in $R e_{K}$ through the use of $u_{*}$ as the relevant velocity scale (Grant et al. 2012), $R e_{K}$ is considered here as the key parameter governing the variation of flow properties across the interface of granular sediment beds.

The strong correlation between $R e_{K}$ and the mass flux across the SWI is demonstrated by the experimental data in figure 1 . Here, the diffusivity that describes mass transport at the SWI $\left(D_{\text {eff }}\right)$ is normalised by the molecular diffusion coefficient of the solute $(D)$ and presented as a function of $R e_{K}$. The data come from the vast dataset of Grant et al. (2012), supplemented by a data point from Roy, Huettel \& Jorgensen (2004) and measurements from Chandler et al. (2016). By assuming a kinematic viscosity of $1 \times 10^{-6} \mathrm{~m}^{2} \mathrm{~s}^{-1}$, curves of constant $R e_{K}$ can be drawn, showing that mass transfer across the SWI is strongly dependent on $\operatorname{Re}_{K}$. For $\operatorname{Re}_{K} \leqslant O(0.01)$, the total rate of mass transport is given by molecular diffusion alone, reflecting the impermeability of the boundary at low $R e_{K}$. Conversely, for $R e_{K} \geqslant O(0.1)$, molecular diffusion becomes a negligible contributor to mass transport at the SWI, indicating that other transport mechanisms must dominate interfacial transfer.

The permeability Reynolds number represents the ratio of the sediment permeability scale to the viscous length scale $v / u_{*}$. A sediment bed of fixed permeability can therefore be considered as either impermeable or permeable, depending on the flow conditions (i.e. the value of $u_{*}$ ). The two limiting conditions of $R e_{K}$ represent (i) an effectively impermeable boundary (i.e. $\left.R e_{K} \ll 1\right)$ and (ii) a highly permeable boundary (i.e. $R e_{K} \gg 1$ ), where turbulence can penetrate into the interstitial fluid (Breugem et al. 2006). As aquatic sediments typically have permeabilities of $10^{-12}-10^{-7} \mathrm{~m}^{2}$ (Rosgen 
(a)

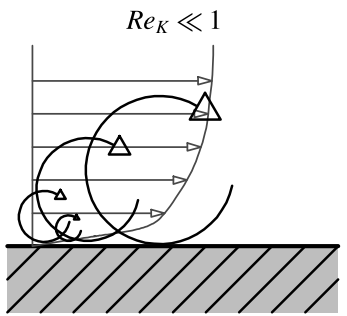

(b)

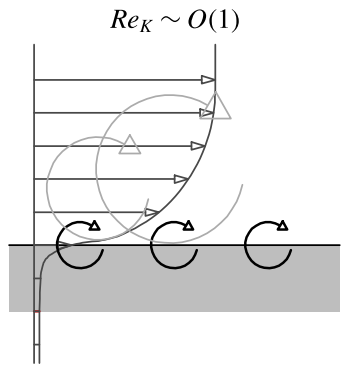

(c)

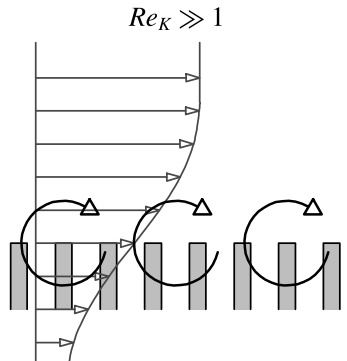

FIGURE 2. Framework identifying flow at the SWI $(b)$ as a transition between flows over impermeable boundaries $(a)$ and highly permeable boundaries $(c)$. The variation of the structure of the mean flow and turbulence with $\operatorname{Re}_{K}$ is shown schematically.

1994; Wilson et al. 2008), and shear velocities of $0.1-10 \mathrm{~cm} \mathrm{~s}^{-1}$, sediment beds in aquatic systems exist in the range $R e_{K}=O(0.001-10)$. This suggests that the sediment bed in aquatic systems is typically located in a range of important transition between the impermeable and fully permeable boundary flow regimes.

\subsection{Flow characteristics for $\operatorname{Re}_{K} \ll 1$}

Flows bounded by an impermeable boundary, either smooth or rough, are characterised by the no-slip condition at the wall and the shear force at the impermeable boundary is given by the sum of the form and the viscous drag of the roughness elements, i.e. $\tau_{R}(z=0)=f_{f}+f_{v}(2.1)$. Further away from the wall, the effects of turbulence grow and turbulent length scales are limited only by the distance from the wall, leading to the classical logarithmic velocity profile (figure $2 a$ ). This depiction is a simplification of the complex nature of turbulence in impermeable boundary layer flows; for example, the viscosity-dominated near-wall region is not completely independent of the effects of turbulent structures in the outer region (e.g. Marusic, Mathis \& Hutchins 2010) and quasi-periodic packets transport low momentum fluid into the outer region and high momentum fluid to the near-wall region (e.g. Adrian, Meinhart \& Tomkins 2000). The impermeability of the wall has a substantial influence on the near-wall transport of energy, as turbulent fluctuations into the wall (i.e. sweeps) are redirected into walltangent components due to the inability of the flow to penetrate the wall. This is referred to as the 'wall-blocking effect' (Perot \& Moin 1995).

\subsection{Flow characteristics for $\operatorname{Re}_{K} \gg 1$}

The interface of a highly permeable boundary connects a region of velocity deficit within the permeable layer to a free stream region above the interface. The interface is characterised by a distinct inflection point in the streamwise velocity profile (figure $2 c$ ) and is typically associated with the presence of a Kelvin-Helmholtz-type (KH) instability (Raupach, Finnigan \& Brunei 1996). This instability leads to the development of coherent turbulent structures with a predictable frequency, corresponding to the natural frequency of instability of the hyperbolic tangent mixing layer velocity profile (Ho \& Huerre 1984). Unlike the broad range of turbulent time and length scales found in the impermeable boundary layer, the interface region is dominated by the single scale of these structures. These structures create much more efficient vertical mixing than those in an impermeable boundary layer flow (Ghisalberti 
\& Nepf 2002; Ghisalberti 2009). As the high permeability allows turbulent structures to penetrate the interface, the transport of mass across the interface is dominated by turbulent transport. Unlike the impermeable boundary layer flow, the interfacial shear force is now dominated by the Reynolds stress, with a small contribution from the form-induced stress $\tau_{R}(z=0) \simeq-\rho \theta\left\langle\overline{u^{\prime} w^{\prime}}\right\rangle-\rho \theta\langle\tilde{u} \tilde{w}\rangle+f_{f}$.

Although the physical appearance of highly permeable media (e.g. urban canopies, submerged vegetation and coral reefs) can vary dramatically, they are all characterised by a drag-induced inflection point in the vertical profile of the mean velocity. The dominance of KH-type turbulent structures in this region engenders a similarity in flows across permeable boundaries at high $R e_{K}$; this similarity is seen in properties such as the depth of penetration of shear into the porous medium, the interfacial velocity $\left(U_{i}=\langle\bar{u}\rangle_{z=0}\right)$ and the turbulence anisotropy at the interface (Ghisalberti 2009). This similarity implies that interfacial properties become independent of permeability for $\operatorname{Re}_{K} \gg 1$. Note, however, that this behaviour does not hold for a continuous increase of the permeability, as the flow typology will eventually revert to that of a classical turbulent impermeable boundary layer (Poggi et al. 2004). For these 'thickness-limited permeable boundaries', where the penetration depth of the flow is constrained by the finite thickness of the permeable medium, other non-dimensional numbers, such as $\sqrt{K} / H_{s}$, become relevant.

\subsection{Flow characteristics for $\operatorname{Re}_{K} \sim O(1)$}

The dynamics of transitional $R e_{K}$ flows have remained largely undocumented as technical limitations complicate the acquisition of detailed flow observations across the interface. Consequently, much of the current understanding comes from experimental measurement of dynamics either above the interface or from numerical simulations. As suggested in figure $2(b)$, the mean velocity profile over a large range of $R e_{K}$ possesses characteristics of both the impermeable boundary and the highly permeable boundary, with a logarithmic velocity profile above the interface (e.g. Suga et al. 2010; Manes, Poggi \& Ridolfi 2011a) and an inflection point at the interface (e.g. Goharzadeh, Khalili \& Jørgensen 2005; Breugem et al. 2006). Similarly to the highly permeable boundary, a shear layer develops below the SWI, reaching a constant mean velocity $\left(U_{p}=\langle\bar{u}\rangle_{z<-\delta_{b}}\right)$ at a distance $\delta_{b}$ below the interface. This depth of penetration is referred to as the Brinkman layer thickness, $\delta_{b}$, a crucial parameter in slip models, and is argued to be proportional to the square root of the permeability or the representative grain diameter, i.e. $\delta_{b} \propto \sqrt{K}$ or $\delta_{b} \propto d$ (e.g. Boudreau 2001; Goyeau et al. 2003; Goharzadeh et al. 2005).

As the permeability of the SWI allows for flow penetration into the porous medium, the interfacial velocity is non-zero. The interfacial velocity at the SWI has been suggested to be a function of the shear velocity $u_{*}$ for $\operatorname{Re}_{K}=O(0.1-1)$ (Suga et al. 2010). Although a clear understanding of the variation of $U_{i} / u_{*}$ with $R e_{K}$ is desirable, the sensitivity of $U_{i}$ to the choice of position of the interface makes determination of $U_{i} / u_{*}$ difficult. For instance, in some studies the SWI is taken at the absolute top of the obstructive elements (e.g. Goharzadeh et al. 2005; Breugem et al. 2006; Suga et al. 2010), which typically corresponds to the definition in highly permeable boundaries (Nepf 2012). However, others adopt a definition closer to that of an impermeable boundary, positioning the interface within the roughness elements (e.g. Nezu \& Nakagawa 1993; Nikora et al. 2001). This links the interface position to the physical length scale of the roughness elements at the interface which, in the case of a flat sediment bed, is the characteristic grain diameter. 
An inflection point in the mean velocity profile, characteristic of highly permeable boundary flows, exists (even at low $R e_{K}$ ) at the SWI (Goharzadeh et al. 2005). Because of this inflection point, linear stability analysis suggests that flow over a porous medium of any non-zero porosity is unstable (White \& Nepf 2007). The existence of these KH-type coherent structures at the SWI at high $R e_{K}$ (where they cannot be damped by viscosity) has been hypothesised by Breugem et al. (2006) and Suga, Mori \& Kaneda (2011) based on two-dimensional snap shots of the interfacial flow field. Experimental evidence of their existence, however, remains sparse (e.g. Manes et al. 2011a), with no experimental evidence for the existence of $\mathrm{KH}$ instabilities at the SWI for granular beds. This suggests that an inflectional velocity profile in these systems is a result of the drag induced by the porous medium and not necessarily indicative of an inflectional instability.

As $R e_{K}$ increases, the flow is able to penetrate the interface, weakening the wall-blocking effect. The relative intensity of the vertical velocity fluctuations therefore increases while the intensity of the streamwise fluctuations decreases (Breugem et al. 2006; Suga et al. 2010; Manes et al. 2011a), enhancing mass transfer (as seen in figure 1). The observed decrease in streamwise turbulence intensity near the interface is most likely related to a change in turbulent structures in the near-wall region, as Breugem et al. (2006) found that the signatures of the large-scale motions, typical for the impermeable boundary, disappear for $R e_{K} \gtrsim 1$. Suga et al. (2011) observed similar changes above the interface for flow over porous foam, where packets of hairpin vortices become unidentifiable at higher values of $R e_{K}$. This suggests that the turbulent structures typically found in impermeable boundary layer flow gradually disappear with increasing $R e_{K}$. The weakening of the wall-blocking effect and the ability of both the mean flow and turbulence to penetrate the interface suggest that none of the terms in (2.1) can be neglected a priori in determining the total shear force (including both fluid stresses and drag at the bed surface) at the interface.

While the framework presented here is consistent with a broad range of studies, it cannot be quantified without experimental measurement of the mean and turbulent flow at the SWI. This experimental study provides these measurements. In this work, we focus on two key issues: (i) demonstrating that the hydrodynamics at the SWI indeed represent a transitional regime that couples two canonical flow typologies (the impermeable boundary and the highly permeable boundary flow) and (ii) that the flow properties at the SWI in this transitional regime are strongly dependent on $R e_{K}$. These observations and this framework are then used to examine the implications for descriptions of interfacial transport in flows over sediment beds.

\section{Methodology}

Laboratory experiments were conducted in a 2-m-long glass-walled flume with a cross-sectional area of $0.4 \times 0.4 \mathrm{~m}^{2}$ (figure 3). Fluid was recirculated by a centrifugal pump and the discharge adjusted by changing the rotational speed of the pump. A false bottom was used at each end of the flume, creating a rectangular void of length $L=1.10 \mathrm{~m}$ and height $H_{S}=0.15 \mathrm{~m}$. The space was randomly filled with monodisperse borosilicate glass spheres to represent a horizontal sediment bed. Sphere diameters of $6 \mathrm{~mm}, 10 \mathrm{~mm}$ and $25 \mathrm{~mm}$ were used (referred to as 'small' (S), 'medium' (M) and 'large' (L), respectively). The depth of fluid above the bed at $x=0(H)$ was kept constant at $9 \mathrm{~cm}$, with a free surface gradient driving the flow. Flow disturbances originating from the inlet were dampened by a combination of porous foam and flow straighteners. The directions of $x, y$ and $z$, with velocity components in those 


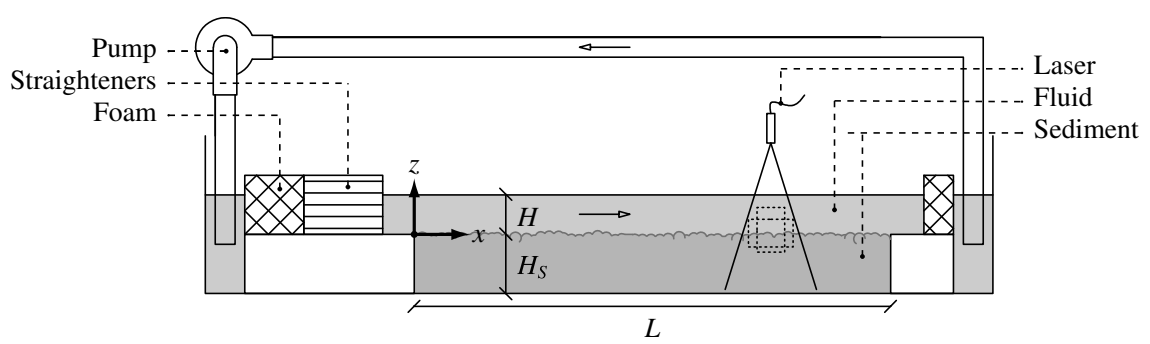

FIGURE 3. Experimental set-up of the recirculating flume. The flow is driven by a centrifugal pump and has a fixed depth of $H=9 \mathrm{~cm}$. The model sediment bed, consisting of monodisperse borosilicate glass beads, has a height $H_{s}=15 \mathrm{~cm}$ and a length $L=1.1 \mathrm{~m}$.

directions given by $u, v$ and $w$, are shown in figure 3 ; the origin is located at the SWI at the leading edge of the sediment bed. The technical difficulties of acquiring detailed measurements across the SWI were overcome here by combining particle tracking velocimetry (PTV) with refractive index matching (RIM).

\subsection{Refractive index matching}

RIM was used here to create an unobstructed view into the interstitial fluid. It has previously been applied to obtain information about the hydrodynamics in obstructed systems, such as at the SWI (Goharzadeh et al. 2005), in canopy flow (Bai, Katz \& Meneveau 2015) and in mechanical systems (Uzol et al. 2002). The fluid chosen was a $59 \%$ by mass sodium iodide (NaI) solution. The fluid had a specific gravity of 1.77 (measured with an Anton Paar DMA500 density meter) and a kinematic viscosity of $1.35 \times 10^{-6} \mathrm{~m}^{2} \mathrm{~s}^{-1}$ (measured with a Cannon-Fenske viscometer tube). Importantly, the fluid had a refractive index of $n=1.4750$ (measured with an Atago pocket refractometer) for a wavelength $(\lambda)$ of $650 \mathrm{~nm}$ at a temperature $(T)$ of $23^{\circ} \mathrm{C}$. This matches the refractive index of borosilicate glass, the material of the model sediment grains. Figure 4 shows the borosilicate glass beads submerged in NaI solutions of increasing concentration: water, $50 \% \mathrm{NaI}$ solution and a $59 \% \mathrm{NaI}$ solution. As light was not refracted when both the fluid and the glass have the same refractive index, an undistorted image under the beads becomes visible in the $59 \%$ solution (figure $4 c$ ). Because the refractive index of the solution is a function of temperature, the fluid temperature was kept at $23.0 \pm 0.8^{\circ} \mathrm{C}$; this ensured that the refractive index of the fluid was maintained within the range $n=1.4750 \pm 0.0002$ (for $\lambda=650 \mathrm{~nm}$ ). Although a yellowing of $\mathrm{NaI}$ solutions due to oxidation has been previously reported, this only creates absorption of the low wavelength range of the visible spectrum, i.e. $\lambda<600 \mathrm{~nm}$ (Uzol et al. 2002; Häfeli et al. 2014), while the laser light used in these experiments had a wavelength of $650 \mathrm{~nm}$.

\subsection{Particle tracking velocimetry}

Two-dimensional instantaneous velocity fields were gathered by means of PTV, a measurement technique successfully applied in similar experiments looking at turbulent boundary layer flow (e.g. Cowen \& Monismith 1997) and flow within a gravel bed pore (Detert et al. 2007). Raw images of illuminated tracer particles in the flow were captured by a CCD (charge-coupled device) camera at a rate of 23 frames per second, where a single frame contains $2448 \times 1200$ px. Two sizes of 
(a)

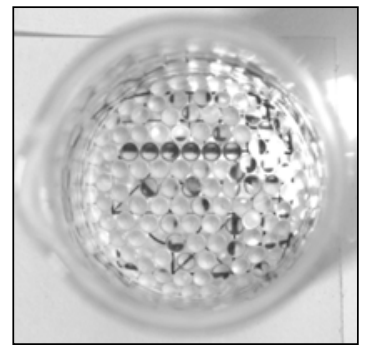

(b)

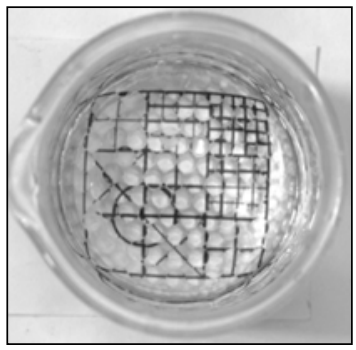

(c)

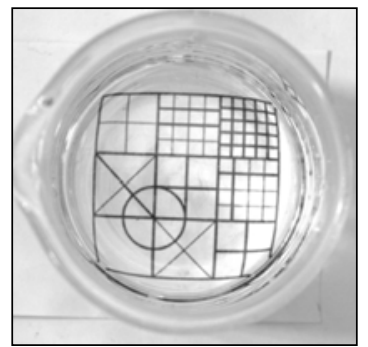

FIGURE 4. The refractive index matching combination of fluid and model sediment used in this study. Shown are borosilicate glass beads in $(a)$ water, $(b) 50 \%$ NaI solution, $(c)$ $59 \% \mathrm{NaI}$ solution. As the refractive index of the glass beads and the fluid are equal in (c), the image underneath the fluid remains undistorted. The $59 \% \mathrm{NaI}$ solution was used in experiments.

$\begin{array}{lccc}\text { Sediment grain diameter }(d)(\mathrm{mm}) & 6 & 10 & 25 \\ \text { Tracer particle diameter }(\mu \mathrm{m}) & 32-40 & 32-40 & 91-100 \\ \text { Field of view }\left(\mathrm{mm}^{2}\right) & 35 \times 70 & 35 \times 70 & 90 \times 180 \\ \text { Laser sheet thickness }(\mathrm{mm}) & 1 & 1 & 3\end{array}$

TABLE 1. Details of the experimental set-up for the different sediment sizes.

custom-made silver coated PMMA (Polymethyl methacrylate) particles were used, with diameters of 32-40 $\mu \mathrm{m}$ and 91-100 $\mu \mathrm{m}$ (and specific gravities of 1.75 and 1.73 , respectively). The imaged diameter of the tracer particles, which is greater than the actual tracer particle diameter, was equivalent to $2.0 \mathrm{px}$, as suggested by Cowen \& Monismith (1997). The tracer particles were illuminated by a 1-3 mm thick laser sheet $\left(650 \mathrm{~nm}\right.$ wavelength) from two aligned $50 \mathrm{~mW}$ lasers with a $60^{\circ}$ fan angle Powell lens. The field of view was approximately $35 \times 70 \mathrm{~mm}$ or $90 \times 180 \mathrm{~mm}$, depending on the size of the tracer particles. The experimental set-up depended on the sediment grain size, as summarised in table 1.

The optimal particle seeding density for PTV is suggested to be 5-20 particles per $32 \times 32$ px (Cowen \& Monismith 1997). This density, however, could not be achieved in our experiments, as tracer particles slowly accumulate in the interstitial fluid and settle on the glass beads. This settling blocks light within the sediments from reaching the camera and hence affects the measurability of the flow field around the SWI. Therefore the seeding density employed was approximately 1 particle per $32 \times 32$ px. The errors due to the lower seeding densities were expected to be small, as PTV is unaffected by displacement gradients (Cowen \& Monismith 1997). The measurements provided, on average, a data point every $4.3 \pm 0.9 \mathrm{~mm}$ for the larger tracer particles and $1.5 \pm 0.2 \mathrm{~mm}$ for the smaller tracer particles. Images were taken over a period of $8.75 \mathrm{~min}$ in each flow and processed in Matlab to enhance contrast and remove the average background. The optimised images were converted to particle fields in the PTV software Streams (Nokes 2016). Coarse velocity fields were first estimated based on the cross-correlation of particle locations in consecutive frames. Subsequently, detailed Lagrangian path fields were determined using the local velocity and acceleration of this coarse velocity field to match individual particles in multiple consecutive frames. The Lagrangian velocity information was then used to determine 


$\begin{array}{lcccccccr}\text { Case } & d(\mathrm{~mm}) & K\left(\mathrm{~mm}^{2}\right) & u_{*}\left(\mathrm{~mm} \mathrm{~s}^{-1}\right) & U_{\delta}\left(\mathrm{mm} \mathrm{s}^{-1}\right) & U_{b}\left(\mathrm{~mm} \mathrm{~s}^{-1}\right) & R e_{K} & R e_{\delta} & R e_{*} \\ \text { S1 } & 6 & 3.96 \times 10^{-2} & 2.44 & 46 & 31 & 0.36 & 868 & 46 \\ \text { S2 } & 6 & 3.96 \times 10^{-2} & 3.18 & 54 & 42 & 0.47 & 1436 & 84 \\ \text { S3 } & 6 & 3.96 \times 10^{-2} & 5.28 & 78 & 62 & 0.78 & 2283 & 154 \\ \text { S4 } & 6 & 3.96 \times 10^{-2} & 6.59 & 104 & 82 & 0.97 & 3171 & 201 \\ \text { M5 } & 10 & 1.10 \times 10^{-1} & 2.93 & 41 & 30 & 0.72 & 1192 & 85 \\ \text { M6 } & 10 & 1.10 \times 10^{-1} & 3.84 & 51 & 39 & 0.94 & 1391 & 104 \\ \text { M7 } & 10 & 1.10 \times 10^{-1} & 4.67 & 62 & 46 & 1.14 & 1867 & 140 \\ \text { M8 } & 10 & 1.10 \times 10^{-1} & 6.04 & 87 & 66 & 1.48 & 2500 & 174 \\ \text { M9 } & 10 & 1.10 \times 10^{-1} & 6.94 & 103 & 78 & 1.70 & 2896 & 196 \\ \text { L10 } & 25 & 6.87 \times 10^{-1} & 1.93 & 31 & 22 & 1.18 & 1310 & 82 \\ \text { L11 } & 25 & 6.87 \times 10^{-1} & 2.78 & 38 & 28 & 1.71 & 1624 & 119 \\ \text { L12 } & 25 & 6.87 \times 10^{-1} & 4.17 & 58 & 41 & 2.56 & 2474 & 178 \\ \text { L13 } & 25 & 6.87 \times 10^{-1} & 6.35 & 86 & 61 & 3.90 & 3701 & 272 \\ \text { L14 } & 25 & 6.87 \times 10^{-1} & 8.09 & 113 & 80 & 4.97 & 4839 & 347 \\ \text { L15 } & 25 & 6.87 \times 10^{-1} & 10.26 & 160 & 115 & 6.30 & 7567 & 536\end{array}$

TABLE 2. Hydrodynamic properties of the experimental cases.

a grid-based Eulerian velocity field by triangular interpolation of the neighbouring tracked particles around a grid point. The grid has a resolution of $0.8 \mathrm{~mm}$ for the 6 and $10 \mathrm{~mm}$ grain diameters and $2.0 \mathrm{~mm}$ for the $25 \mathrm{~mm}$ grain diameter. This is of the same order as the tracer particle spacing but an order of magnitude smaller than the grain diameters.

\subsection{Experimental conditions}

An overview of the hydrodynamic conditions of the experiments is given in table 2, where the bulk velocity is defined as $U_{b}=(1 / \delta) \int_{0}^{\delta} \theta\langle\bar{u}\rangle \mathrm{d} z$. Three different sediment sizes were used; with the variation of the shear velocity $u_{*}$, this allowed a large experimental range of $R e_{K}$. The permeability of the sediments $K$ was estimated using the Carman-Kozeny model:

$$
K=\frac{\theta_{p}^{3}}{180\left(1-\theta_{p}\right)^{2}} d^{2},
$$

where $d$ is the grain diameter. The porosity $\theta_{p}$ was determined by measuring the volume of the interstitial fluid in the flume, yielding $\theta_{p}=0.41$ for all three cases. Although the diameters of the sediments do not correspond to typical sediment sizes in aquatic systems, the range of $R e_{K}$ in these experiments was $0.36-6.3$ (table 2), which is typical of the values for sediments in real aquatic systems.

As the sediment bed had a finite length, it was necessary to determine the point beyond which there was no further evolution of the mean and turbulent velocity flow structure at the SWI. To determine this point, 11 PTV measurements along a streamwise transect were taken in case M7 (table 2). The flow statistics $20 \mathrm{~mm}$ above the SWI along this transect are shown in figure 5, where they are normalised by the spatially averaged statistics in the range $700<x<800 \mathrm{~mm}$ (where $x=0$ corresponds to the leading edge of the sediment bed). The statistics are horizontally averaged over intervals of $30 \mathrm{~mm}$. The streamwise velocity $\langle\bar{u}\rangle$, the turbulence intensities 


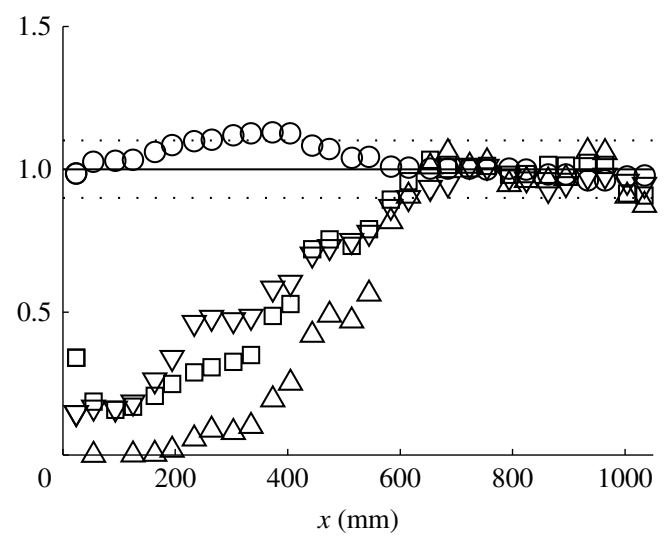

FIGURE 5. Flow statistics along a longitudinal transect $20 \mathrm{~mm}$ above the SWI: $\langle\bar{u}\rangle(\bigcirc)$, $\sigma_{u}(\square), \sigma_{w}(\nabla),\left\langle\overline{u^{\prime} w^{\prime}}\right\rangle(\triangle)$. The statistics are normalised by the average value in the range $700<x<800 \mathrm{~mm}$, where $x=0$ corresponds to the leading edge of the sediment bed. Fully developed flow at the interface is reached by $x=600 \mathrm{~mm}$.

$\sigma_{u}=\left\langle\overline{u^{\prime 2}}\right\rangle^{1 / 2}$ and $\sigma_{w}=\left\langle\overline{w^{\prime 2}}\right\rangle^{1 / 2}$ and the Reynolds stress $\left\langle\overline{u^{\prime} w^{\prime}}\right\rangle$ along this transect do not change beyond $x=600 \mathrm{~mm}$ (figure 5). Therefore, the measurement location was centred around $x=750 \mathrm{~mm}$, by which point there is no further evolution of the interfacial flow. At this position, the boundary layer thickness $\delta$ for case M7 was measured as $41 \mathrm{~mm}$, which is reasonably close to the predicted flat plate value of $35 \mathrm{~mm}$ (see Schlichting (1979, p. 638)). This small difference in $\delta$ can be explained by the destabilising effect of wall permeability (Tilton \& Cortelezzi 2008), which allows a wall-normal velocity component at the interface.

Due to the strong spatial variability of flows over porous media, measurements were taken at three lateral positions to obtain horizontally averaged statistics of the flow properties across the SWI. The lateral measurement positions were at $65 \mathrm{~mm}, 95 \mathrm{~mm}$ and $115 \mathrm{~mm}$ from the centreline of the flume (closer to the flume wall nearer the camera). Six measurements of the flow field were recorded in each case. At each of the three lateral positions the camera frame was orientated: (i) horizontally in the $x-z$ plane (to maximise the streamwise distance over which flow statistics were averaged) and (ii) vertically in the $x-z$ plane (to fully capture the boundary layer) (figure 3). Horizontally averaged flow statistics at any position were obtained by averaging across these six measurements, regardless of the camera's orientation. The uncertainty of these spatially averaged statistics is dominated by the finite number of laterally spaced measurements and is taken as the standard error of the laterally spaced estimates. Uncertainties for the spatially and time-averaged flow statistics at the SWI were found to be limited to $5 \%$ for $\langle\bar{u}\rangle, 3 \%$ for both $\sigma_{u}$ and $\sigma_{w}$, and $30 \%$ for $\left\langle\overline{u^{\prime} w^{\prime}}\right\rangle$ (although limited to $6 \%$ for cases S3-L15). This larger uncertainty in $\left\langle\overline{u^{\prime} w^{\prime}}\right\rangle$ for cases S1 and $\mathrm{S} 2$ is due to the low Reynolds stresses in those cases.

\subsection{Critical definitions of the interface and flow properties}

Interpretation of the interfacial flow properties, such as the mean interfacial velocity and the bed shear stress, is strongly dependent on the definition of the SWI. For a rough boundary, the position of the interface is generally taken as 0.15-0.30 $k_{s}$ below the absolute top of the roughness elements (Nezu \& Nakagawa 1993), 

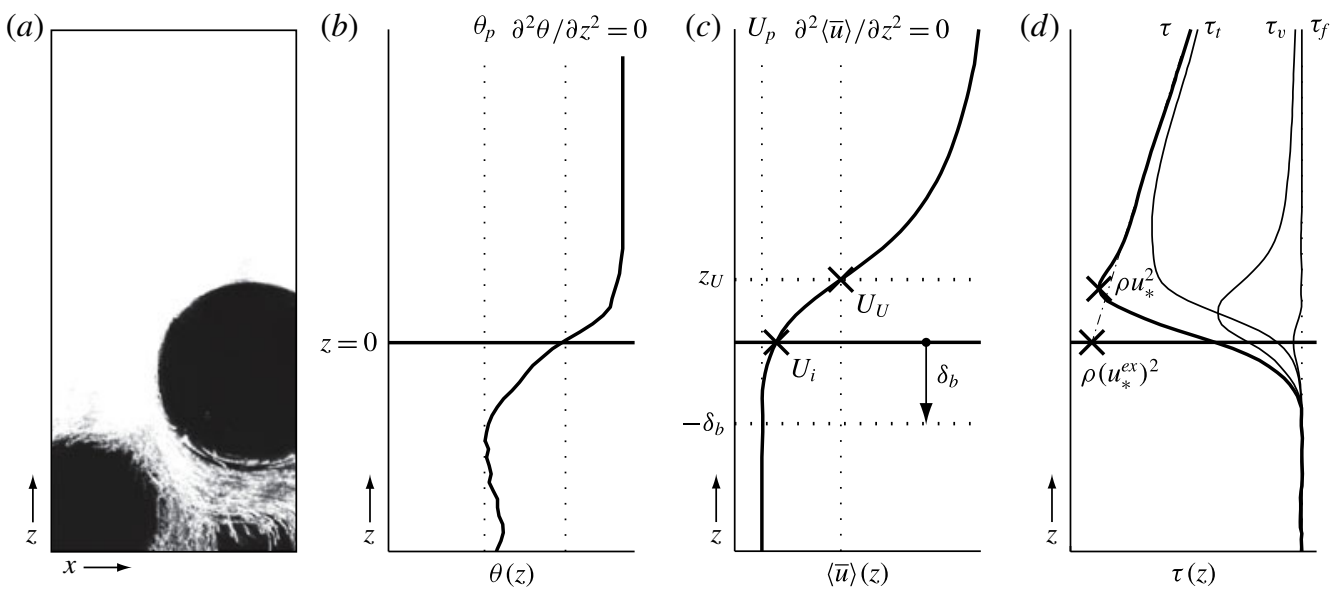

FIgURE 6. (a) A segment of a long exposure image in Case M6; (b) a profile of porosity $(\theta)$. The inflection point defines the position of the SWI $(z=0) ;(c)$ the spatially averaged velocity profile, which defines the Brinkman layer thickness $\delta_{b}$, the constant velocity below the Brinkman layer $U_{p}$, the interfacial velocity $U_{i}$ and the inflectional velocity $U_{U} ;(d)$ summation of fluid stresses $(\tau)$ and its individual shear stress components: the turbulent shear stress $\left(\tau_{t}\right)$, the viscous shear stress $\left(\tau_{v}\right)$ and the form-induced shear stress $\left(\tau_{f}\right)$, where the maximum value of $\tau$ defines the shear velocity $u_{*}$. The absolute top of the sediment bed and the inflection point in the mean velocity profile are coincident. The vertical distance between the inflection points in the velocity and porosity profiles, i.e. $z_{U}$, corresponds to approximately $0.3 d$.

where $k_{s}$ is the length scale of the dominant roughness elements at the boundary (and corresponds to the grain diameter $d$ in a monodisperse sediment bed). Other interface positions considered include the absolute top of the roughness elements (e.g. Goharzadeh et al. 2005; Breugem et al. 2006) and the average bed elevation (e.g. Nikora et al. 2001; Mignot et al. 2009). As the measurement technique in our experimental set-up allows for determination of the porosity profile by measuring the area occupied by the sediments (figure $6 a$ ), the interface has been defined here as the position of the inflection point of the spatially averaged porosity profile (figure $6 b$ ), a definition similar to the average bed elevation. The distance between the porosity inflection point and the absolute top of the sediment bed is equal to $0.3 d$ here, within the range given by Nezu \& Nakagawa (1993). The absolute top of the sediments typically corresponds to the location of the inflection point of the velocity profile (figure $6 c$ ), a distance $z_{U}$ above the interface (Nikora et al. 2004).

The Brinkman layer thickness $\left(\delta_{b}\right)$ is defined similarly to the boundary layer thickness, and is taken as the vertical distance between the SWI $(z=0)$ and the point at which the difference between the local mean velocity and $U_{p}$ has decayed to $1 \%$ of the interfacial value (i.e. $\left.\langle\bar{u}\rangle_{z=-\delta_{b}}=0.01\left(U_{i}-U_{p}\right)+U_{p}\right)$. Note that, hereafter, subscripts ${ }_{i}$ and $U$ are used to denote properties at the interface and the velocity inflection point respectively (as shown in figure $6 c$ ).

Although the bed shear stress at the interface is theoretically defined for both the impermeable and the permeable boundary (Nikora et al. 2001), a broad range of definitions for shear velocity in rough bed flows exists due to the challenges that come with determining $u_{*}$ experimentally (e.g. Pokrajac et al. 2006). As the absence of free surface level measurements prevents the description of $u_{*}$ based on a control 

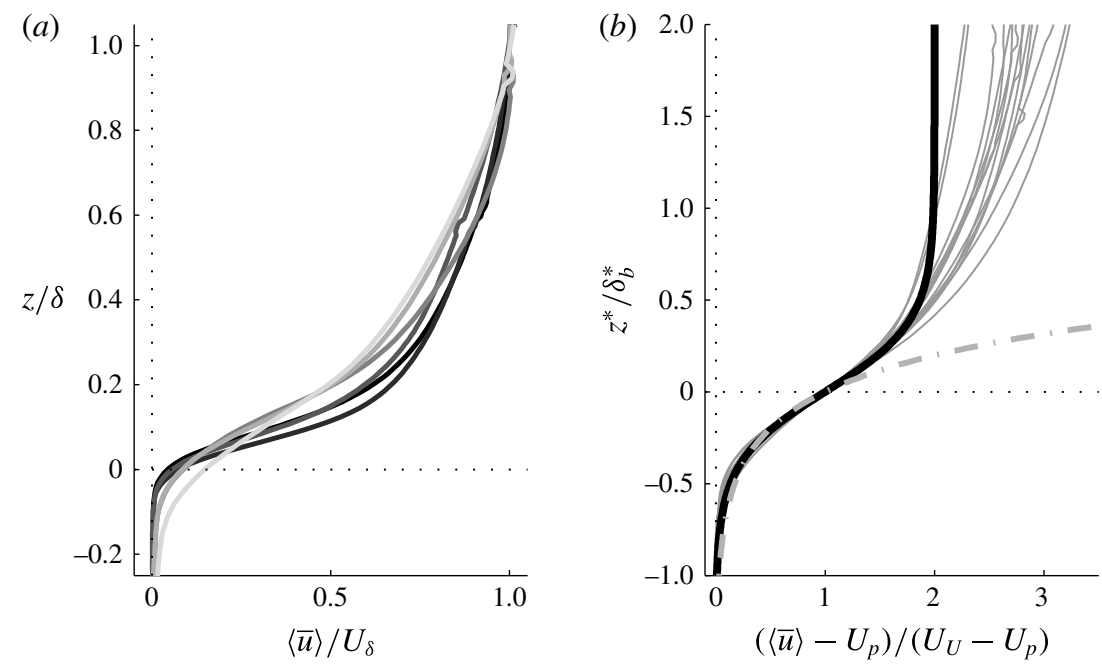

Figure 7. (a) Mean velocity profiles for six cases (S2, S3, M7, L11, L12, L14; from black to grey respectively, where lines become lighter for increasing $R e_{K}$ ), with lengths and velocities normalised by outer scales (i.e. the boundary layer thickness, $\delta$, and the outer velocity, $\left.U_{\delta}\right)$. (b) Velocity profiles for all cases with velocity and length scales normalised by the inflectional velocity $U_{U}$ and the extended Brinkman layer thickness $\delta_{b}^{*}$. The grey lines represent profiles from all experimental cases while the best fit hyperbolic tangent profile and exponential profile to the experimental data below $z^{*}=0$ are given in black and dashed grey respectively.

volume force balance, the only representative definition of $u_{*}$ is through calculation of the maximum total fluid shear stress as, below this point, drag from the roughness elements will contribute to the flow resistance. Hence, the shear velocity is defined as $u_{*}=\sqrt{\tau_{\max } / \rho}$ (figure $6 d$ ), where $\tau_{\max }$ stems from (2.1) (noting that $f_{f}$ and $f_{v}$ are zero above the roughness crests) and is positioned vertically at $z=(0.5 \pm 0.2) d$. As this definition of $u_{*}$ (consistent with that of Pokrajac et al. 2006) provides a dynamically relevant measure of shear stress, it has been adopted here. An alternative definition is based on the extrapolation of the linear total fluid shear stress profile in the turbulent boundary layer to the interface, which yields $u_{*}^{e x}$ (figure $6 d$ ). Our results suggests that the difference is small $\left(1.04<u_{*}^{e x} / u_{*}<1.16\right)$, such that the interpretation of the data in this study is insensitive to the choice of definition of shear velocity.

\section{Results}

\subsection{Mean velocity structure}

The mean velocity profiles across the SWI for six cases are shown in figure 7(a), where length and velocity scales are normalised by the outer scales ( $\delta$ and $U_{\delta}$, respectively). All profiles are characterised by: (i) an inflection point above the SWI, (ii) a non-zero interfacial velocity and (iii) flow penetration beyond the interface, all established characteristics for flows over highly permeable boundaries. Note, however, that both the normalised flow penetration depth and the interfacial velocity monotonically increase with $R e_{K}$, leading to differences in detail in the profiles.

The scaling of the velocity profiles by the outer scales does not collapse all profiles above the SWI. An alternative method of examining the profiles is through 


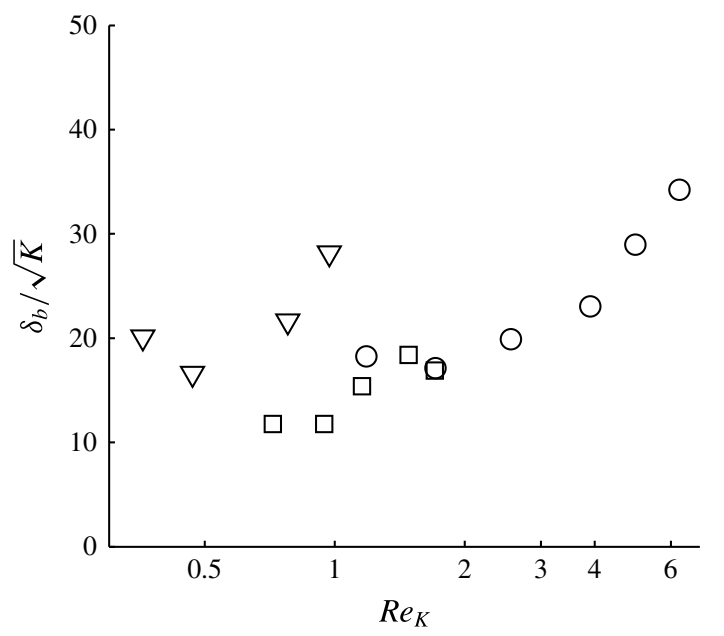

FigURE 8. The $R e_{K}$ dependence of the Brinkman layer thickness $\delta_{b}$ normalised by $\sqrt{K}$ : $d=6 \mathrm{~mm}(\nabla) ; d=10 \mathrm{~mm}(\square) ; d=25 \mathrm{~mm}(\bigcirc)$. The generally accepted proportionality of $\delta_{b} \propto \sqrt{K}$ is not fully observed here, with $\delta_{b} / \sqrt{K}$ increasing with $R e_{K}$.

non-dimensionalisation by the inner scales (Goharzadeh et al. 2005; Breugem et al. 2006; Manes et al. 2011a), here formulated as $\delta_{b}^{*}=\delta_{b}+z_{U}$ (i.e. the vertical distance between the top of the roughness and the bottom of the Brinkman layer) and the inflectional velocity $U_{U}$. In this analysis, the vertical coordinate origin is shifted to the inflectional point of the velocity profile (i.e. $z^{*}=z-z_{U}$ ). Normalisation of the velocity profiles by these inner variables reveals a collapse of the data below $z^{*}=0$ (figure $7 b$ ), suggesting that the inner variables are of importance across the SWI. The collapsed velocity profiles in the Brinkman layer can be described by either a hyperbolic tangent profile, $\left(\langle\bar{u}\rangle-U_{p}\right) /\left(U_{U}-U_{p}\right)=1+\tanh 2.6 z^{*} / \delta_{b}^{*}\left(R^{2}=0.99\right)$, or an exponential relation, $\left(\langle\bar{u}\rangle-U_{p}\right) /\left(U_{U}-U_{p}\right)=\exp \left(3.5 z^{*} / \delta_{b}^{*}\right)\left(R^{2}=0.97\right)$ (figure $\left.7 b\right)$. The hyperbolic tangent function has a superior fit to the experimental data below $z^{*} / \delta_{b}^{*} \approx-0.25$.

The thickness of the Brinkman layer has traditionally been described as depending on the permeability of the sediments only (e.g. Boudreau 2001; Goyeau et al. 2003; Goharzadeh et al. 2005). The results, however, do not fully support a linear proportionality of the Brinkman layer thickness to $\sqrt{K}$, and suggest a correlation with $\operatorname{Re}_{K}$ (figure 8) (although separation of behaviour between cases of different sediment size is apparent). As $\operatorname{Re}_{K} \rightarrow 0$, the penetration depth does not approach zero and retains a finite size, here between $(10-20) \sqrt{K}$. The distance between $z=0$ and the minimum in the porosity profile is equal to $(17.8 \pm 3.3) \sqrt{K}$, suggestive of an influence of the inhomogeneous porosity in governing momentum penetration into the sediment.

\subsection{Turbulence structure}

The permeability of the sediment influences the structure of turbulence above the interface and is associated with a weakening of the wall-blocking effect. Above the SWI, increasing $R e_{K}$ leads to an increase in the vertical turbulence intensity and a decrease in the streamwise turbulence intensity (figure 9). At and below the SWI, 

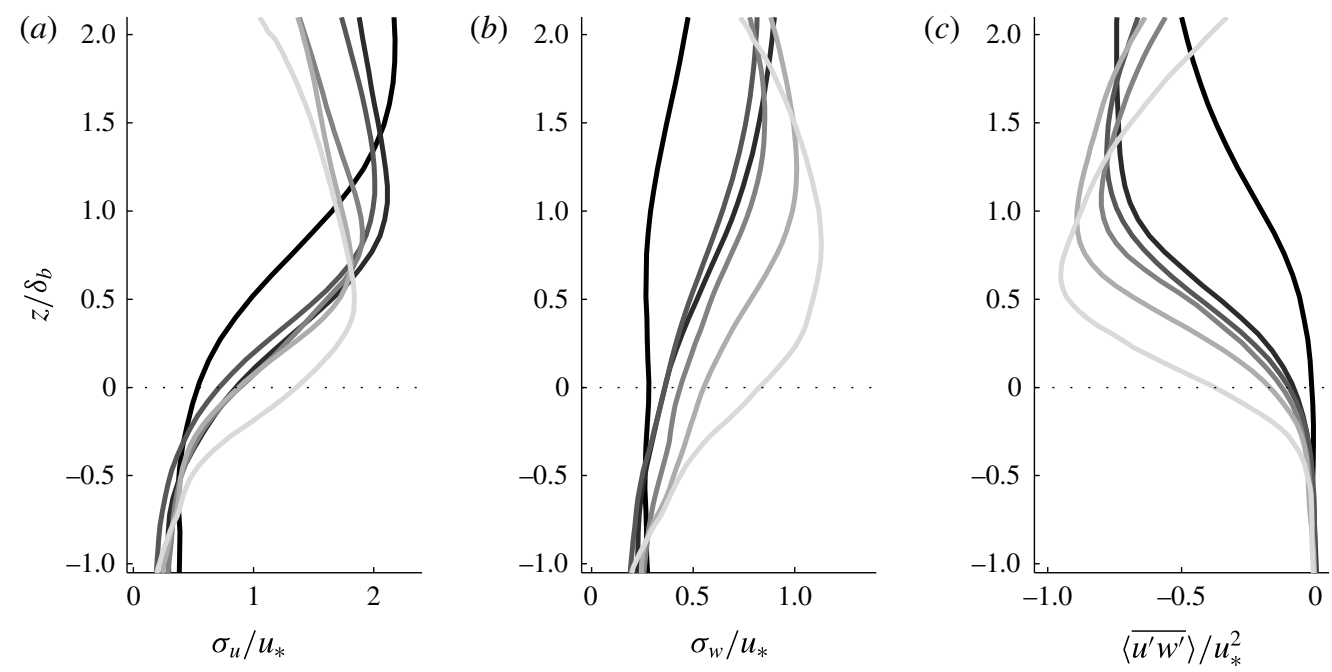

FIGURE 9. Turbulence statistics for six cases (S2, S3, M7, L11, L12, L14; from black to grey respectively) with varying $R e_{K}$ : (a) streamwise turbulence intensity, $(b)$ vertical turbulence intensity, $(c)$ Reynolds stress. Vertical distance is normalised by the Brinkman layer thickness $\left(\delta_{b}\right)$ and turbulent velocities by the shear velocity $\left(u_{*}\right)$. The normalised turbulence intensities and the turbulent shear stress at the SWI increase monotonically with $R e_{K}$.

both the streamwise and vertical turbulence intensities increase with $\operatorname{Re}_{K}$, reflecting the ability of turbulence to penetrate the sediment bed. The turbulent transport of momentum across the SWI can be significant and, in the case of $R e_{K}=4.97$, $\left\langle\overline{u^{\prime} w^{\prime}}\right\rangle_{i}=0.38 u_{*}^{2}$.

The interfacial form-induced stresses are smaller than the turbulent stresses for all $\operatorname{Re}_{K}$ (figure 10). Normalised peak values for all stresses correspond qualitatively to those found by Nikora et al. (2001), except for cases L11 and L12 where both the vertical form-induced intensity and the form-induced shear stress are larger. For both $\left\langle\tilde{w}^{2}\right\rangle^{1 / 2} / u_{*}$ and $\langle\tilde{u} \tilde{w}\rangle / u_{*}^{2}$ peak values and interface values increase with $R e_{K}$. The streamwise stress $\left\langle\tilde{u}^{2}\right\rangle^{1 / 2} / u_{*}$, however, seems uncorrelated to $R e_{K}$, and shows a rather consistent profile across the interface. The vertical positions of maxima in $\left\langle\tilde{u}^{2}\right\rangle^{1 / 2} / u_{*}$ and $\left\langle\tilde{w}^{2}\right\rangle^{1 / 2} / u_{*}$ are distinctly different, being above the interface for $\left\langle\tilde{u}^{2}\right\rangle^{1 / 2} / u_{*}$ (focused around the absolute top of the sediments) and predominantly below the interface for $\left\langle\tilde{w}^{2}\right\rangle^{1 / 2} / u_{*}$. The form-induced shear stress is typically much smaller than the turbulent shear stress, although both are of the same order of magnitude in the lower half of the Brinkman layer.

At the SWI, the viscous stress and the turbulent stress are dominant contributors to the total fluid shear stress (figure 6). The relative contribution of these stresses is shown in figure 11. For $R e_{K} \rightarrow 0, \tau_{t}$ is small compared to $\tau_{v}$, while for $R e_{K}>4$, $\tau_{t}$ dominates over $\tau_{v}$. At the threshold $R e_{K} \approx 1-2$, the two stresses are comparable in magnitude. These findings suggest that the DBL model is inapplicable to porous media at high $R e_{K}$.

The penetration depth of the turbulent shear stress into the bed $\left(\delta_{p}\right)$, defined as $\left\langle\overline{u^{\prime} w^{\prime}}\right\rangle_{z=-\delta_{p}}=0.01\left\langle\overline{u^{\prime} w^{\prime}}\right\rangle_{i}$, increases with $\operatorname{Re}_{K}$ (figure 12a). Although direct proportionality between $\delta_{p} / \sqrt{K}$ and $R e_{K}$ (as proposed by Manes et al. 2012) is not observed 

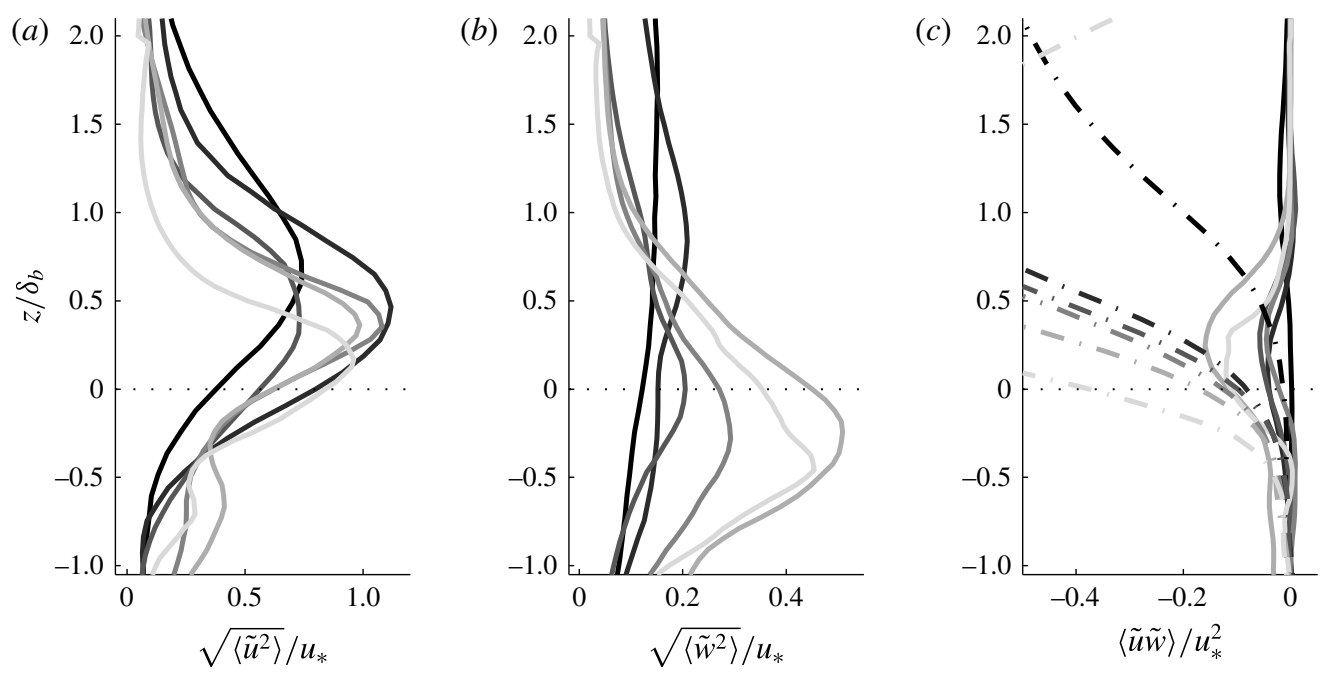

FIGURE 10. Form-induced intensities for six cases (S2, S3, M7, L11, L12, L14; from black to grey respectively) with varying $\operatorname{Re}_{K}$ : (a) streamwise form-induced normal stress, (b) vertical form-induced normal stress, $(c)$ form-induced shear stress (solid), Reynolds stress (dashed). Vertical distance is normalised by the Brinkman layer thickness $\left(\delta_{b}\right)$ and stresses by the shear velocity. Above the interface, the form-induced shear stress is typically much smaller than the turbulent shear stress.

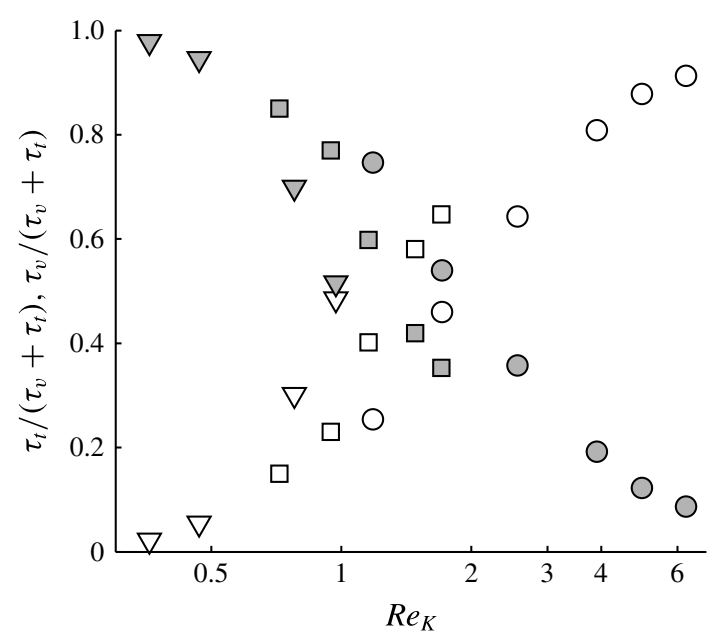

FIgURE 11. Partitioning of the fluid shear stress at the SWI as a function of $R e_{K}: d=$ $6 \mathrm{~mm}(\nabla) ; d=10 \mathrm{~mm}(\square) ; d=25 \mathrm{~mm}(\bigcirc)$. The individual stress components, viscous stress $\tau_{v}$ (grey) and turbulent stress $\tau_{t}$ (white), are normalised by their sum. The turbulent stress is negligible at low $R e_{K}$, but exceeds the viscous stress above $R e_{K} \simeq 1-2$.

for the $R e_{K}$ range examined here, this study indeed suggests a dependence of $\delta_{p} / \sqrt{K}$ on $\operatorname{Re}_{K}$ (figure $12 a$ ). Contrary to the penetration of mean velocity, the turbulent shear penetration approaches zero in the limit of $R e_{K} \rightarrow 0$. When the penetration depths of mean velocity and turbulent shear stress from the absolute top of the sediment 

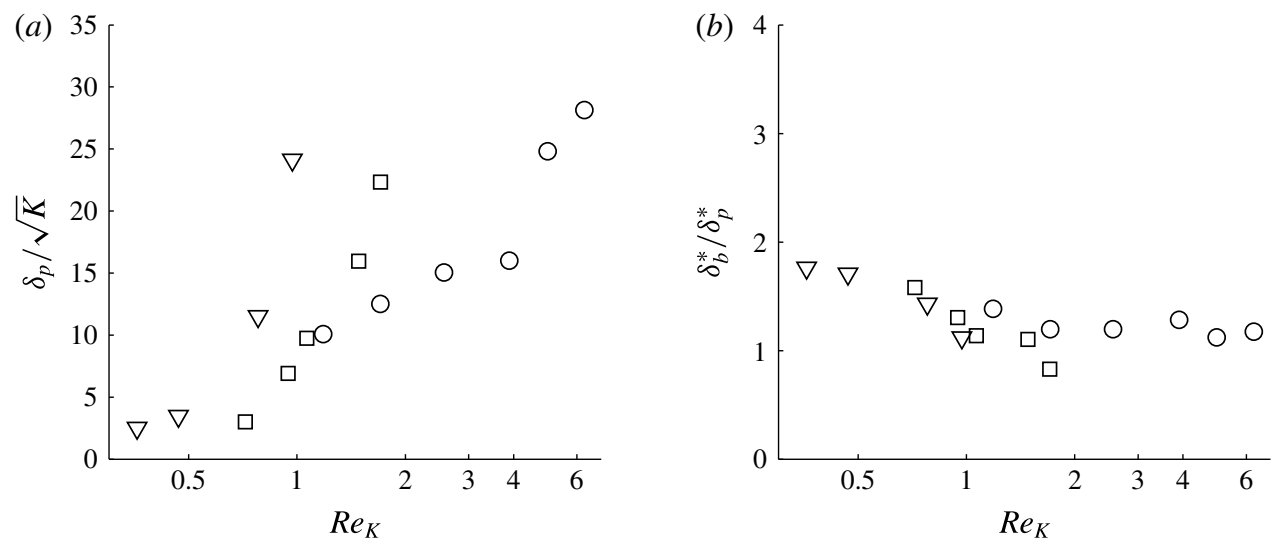

FIgURE 12. (a) The increase of the normalised shear penetration depth $\left(\delta_{p} / \sqrt{K}\right)$ with $R e_{K}: d=6 \mathrm{~mm}(\nabla) ; d=10 \mathrm{~mm}(\square) ; d=25 \mathrm{~mm}(\bigcirc)$. (b) Comparison of the shear stress penetration depth to the flow penetration depth, both relative to $z_{U}$, suggesting transitional behaviour above $\operatorname{Re}_{K} \simeq 1-2\left(\delta_{b}^{*} / \delta_{p}^{*} \simeq 1.25\right)$.

bed (i.e. $\delta_{p}^{*}=\delta_{p}+z_{U}$ ) are compared, a deterministic relationship is apparent, with transitional behaviour above $R e_{K} \simeq 1-2$ of $\delta_{b}^{*} / \delta_{p}^{*} \simeq 1.25$ (figure $12 b$ ). Hence, beyond this threshold, the mean flow penetration depth is directly proportional to the shear penetration depth. The similar increases with $R e_{K}$ of turbulent transport from the water column to the sediments (figure 11) and the penetration of momentum into the sediment $\left(\delta_{p}\right.$, figure 12a) suggest that the growth of the Brinkman layer is caused by an increased penetration of turbulent structures into the sediment.

\subsection{Similarity of flows at high $\mathrm{Re}_{K}$}

Flows over highly permeable boundaries are thought to share statistical flow similarities, despite the wide range of permeable medium geometries (Ghisalberti (2009), herein referred to as G09). The similarity relations in G09 are given as follows:

$$
\begin{aligned}
& \frac{\sigma_{w}}{\sigma_{u}} \simeq 0.6, \\
& \frac{\sigma_{u}}{u_{*}} \simeq 1.8, \\
& \frac{\sigma_{w}}{u_{*}} \simeq 1.1, \\
& \frac{U_{i}}{u_{*}} \simeq 2.6,
\end{aligned}
$$

where all the properties are defined at the interface of a highly permeable boundary, i.e. the interface is positioned at $z_{U}$ and $u_{*}$ is based on the turbulent shear stress alone. The relations (4.1)-(4.4) are compared to our experimental results based on two limiting case normalisation schemes: the first based on the framework of the impermeable boundary and the second on the highly permeable boundary. The distinction is necessary as typologies at both limits of $R e_{K}$ define parameters, notably the position of the interface and the shear velocity, fundamentally differently. 
In figure $13(a, c, e, g)$, flow properties are taken at $z=0$, in accordance with the impermeable limit. As the interface of highly permeable boundaries typically corresponds to the inflection point of the mean velocity profile, properties in figure $13(b, d, f, h)$ are taken at $z_{U}$. Note that the normalisation of velocity scales in flows over highly permeable boundaries is typically based on the Reynolds stress at $z_{U}$ (i.e. normalisation by $\left(-\left\langle\overline{u^{\prime} w^{\prime}}\right\rangle_{U}\right)^{1 / 2}$ ). This normalisation procedure demonstrates the similarity to impermeable boundaries as $R e_{K} \rightarrow 0$ on the left-hand side, and similarity to highly permeable beds as $\operatorname{Re}_{K} \rightarrow \infty$ on the right-hand side of figure 13 .

The ratio of the turbulence intensities $\sigma_{w} / \sigma_{u}$ is a measure of the turbulence anisotropy and maintains a value of about 0.6 at the interface of highly permeable boundaries. For a smooth impermeable boundary, the anisotropy approaches zero (Kim, Moin \& Moser 1987) due to the wall-blocking effect. The anisotropy at the SWI for these experiments (figure $13 a$ ) shows a relatively constant ratio with varying $R e_{K}$, without any signs of a trend towards the low $R e_{K}$ limit. The absence of enhanced anisotropy for low $R e_{K}$ is possibly caused by the presence of recirculation zones in the interstitial volumes, which can decrease the turbulence anisotropy (Smalley et al. 2002). The anisotropy at the inflection point, however, shows a significantly different trend (figure 13b), but is still consistent with the weakening of the wall-blocking effect above the interface, which results in a lower ratio of $\sigma_{w, U} / \sigma_{u, U}$ with decreasing $R e_{K}$. While the trend for $R e_{K} \rightarrow 0$ corresponds to the low $R e_{K}$ limit, the trend suggested by the experimental data does not suggest an asymptotic relation for a further increase in $R e_{K}$. The anisotropy at the inflection point is, however, strongly correlated to $R e_{K}$, and suggests an increasing relevance of turbulent structures at $z_{U}$ with increasing relative permeability.

A gradual change in the interaction between the SWI and turbulent structures in the flow can be observed from the turbulence intensities across the SWI (figure 13c- $f$ ). They show a steady progression of the turbulence intensities with $R e_{K}$ at the SWI, and a decreasing streamwise turbulence intensity at $z_{U}$, consistent with a weakening of the wall-blocking effect. Although evidence for these mechanisms was seen in figure 9, the trends in figure $13(c-f)$ show a steady dependence of the turbulence intensities on $R e_{K}$, suggesting impermeable boundary behaviour for $R e_{K} \rightarrow 0$ where the turbulence intensities at the interface approach zero (figure $13 c, e$ ), and a tendency towards flows over highly permeable boundaries with increasing $\operatorname{Re}_{K}$ (figure $13 d, f$ ).

The mean velocity at the interface shows a strong correlation with $\operatorname{Re}_{K}$ (figure $13 g, h$ ). The data suggest that the interfacial velocity can be larger than zero for an impermeable boundary, consistent with the non-zero Brinkman layer thickness for the same limit. For the inflectional velocity, an asymptotic limit in $R e_{K}$ is suggested of $U_{U} /\left(-\left\langle\overline{u^{\prime} w^{\prime}}\right\rangle_{U}\right)^{1 / 2} \sim 6.5$ for $R e_{K}>O(1)$. This relation is however larger than the 2.6 value suggested in G09.

\section{Discussion}

Our hydrodynamic framework and the use of a novel experimental approach provides a description of the nature of interaction between permeable sediments and the overlying flow. This framework places flow at the SWI in the context of two extreme flow typologies, impermeable boundary layer flow and flow over highly permeable boundaries, through consideration of the dependence of the flow on $R e_{K}$. As typical aquatic sediments correspond to moderate values of $R e_{K}$, elements of the hydrodynamic characteristics of both extremes can be seen in the flow at the SWI. The experimental data describe the flow across the SWI for a broad range of $R e_{K}$ relevant to aquatic systems. 

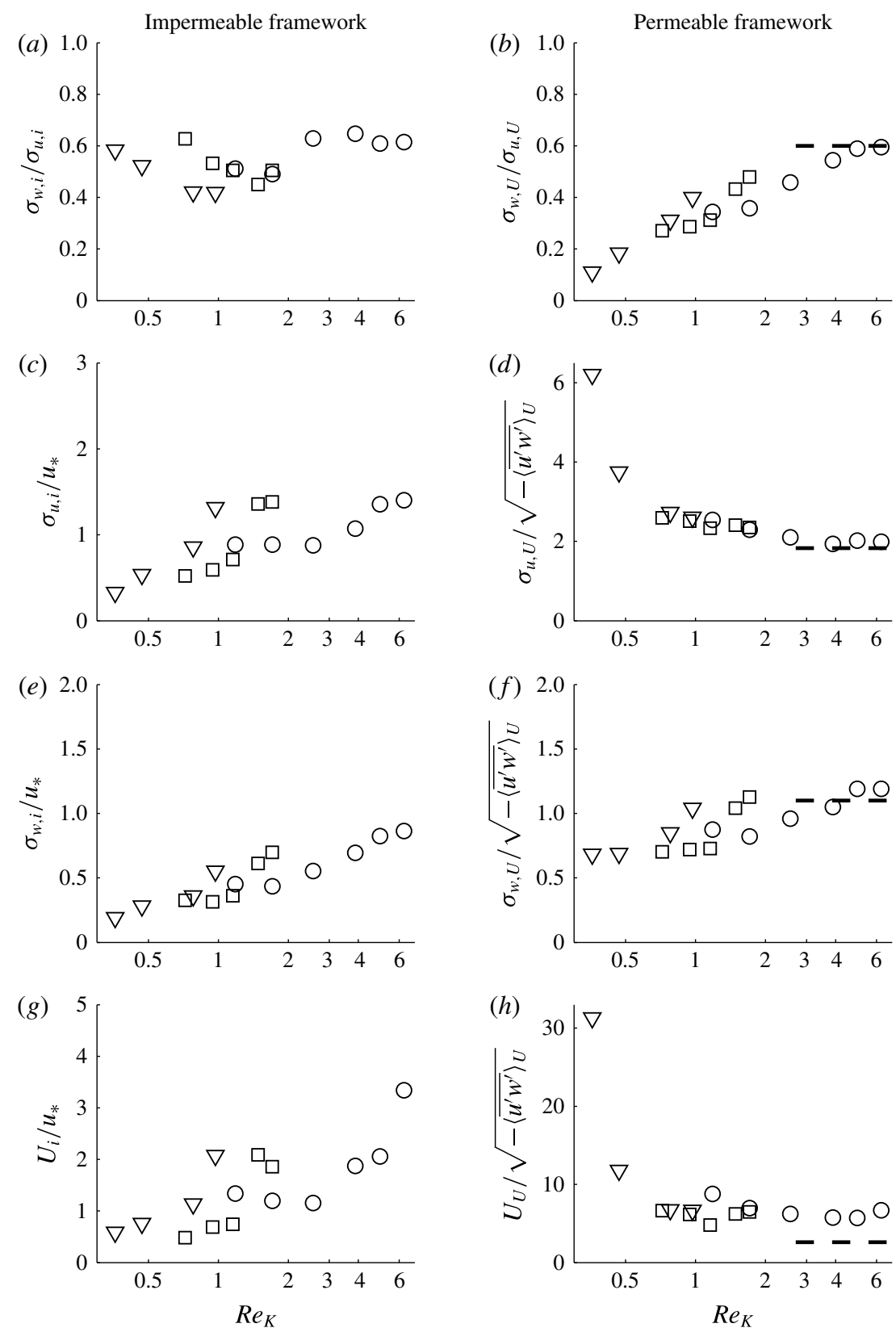

FIGURE 13. Four similarity relations for flow at the SWI which, according to the framework, should display asymptotic behaviour as $R e_{K} \rightarrow \infty$ (given by (4.1)-(4.4)): $d=6 \mathrm{~mm}(\nabla) ; d=10 \mathrm{~mm}(\square) ; d=25 \mathrm{~mm}(\bigcirc)$. These limits are indicated by dashed lines, and are given in Ghisalberti (2009). The relations are presented in the framework of the impermeable and highly permeable boundaries. For the former, presented on the left-hand side $(a, c, e, g)$, data are taken at the SWI $(z=0)$ and normalised by $u_{*}$. For the latter, presented on the right-hand side $(b, d, f, h)$, data are taken at the velocity inflection point $\left(z=z_{U}\right)$ and normalised by $\left(-\left\langle\overline{u^{\prime} w^{\prime}}\right\rangle_{U}\right)^{1 / 2}$, which is conventional for flows over highly permeable boundaries. The data show good agreement with the impermeable limits for $R e_{K} \rightarrow 0$ in the left-hand side panels and good agreement with the highly permeable limits for $R e_{K} \rightarrow \infty$ in the right-hand side panels. 
The results presented here confirm the strong correlation between interfacial flow properties and the relative permeability, where key properties such as the Brinkman layer thickness, shear stress penetration depth, the interfacial turbulence intensities, turbulent shear stress and mean velocity all increase with $R e_{K}$. Values in the limit of $R e_{K} \rightarrow 0$ correspond to values typically expected for impermeable boundary layer flow, with the exception of the interfacial velocity and Brinkman layer thickness, which is argued to be the consequence of a heterogeneous interface region.

At the high $R e_{K}$ limit, the properties at the inflection point in the velocity profile are consistent with the relations suggested in G09 for flows over highly permeable boundaries. Our data suggest that all flows at the SWI possess an inflectional velocity profile. An observation consistent with the results of Goharzadeh et al. (2005) and Breugem et al. (2006), however, inconsistent with the results of Nikora et al. (2001) and Mignot et al. (2009). Given that the velocity profile below the interface (i.e. over the Brinkman layer) matches that of a hyperbolic tangent profile, the mean streamwise velocity at and below the interface is consistent with that in flows over a highly permeable boundary.

Flows over sediments are, however, still typically treated as impermeable. While the region around the SWI $\left(z=\left[-\delta_{b}, z_{U}\right]\right)$ is small compared to the boundary layer thickness, it is exactly this region where the flow and sediment properties influence the interfacial transport processes. Our results suggest that this region invariably shows similarities to that of a highly permeable boundary, with the penetration of momentum across the interface and the presence of an inflectional velocity profile. Beyond $\operatorname{Re}_{K} \sim 1-2$, transition is observed for key interfacial flow characteristics towards the asymptotic limits described for highly permeable boundaries, for instance the penetration of the turbulent shear stress. These similarities with highly permeable boundaries suggest a change in our perspective on flows over porous media; namely for $R e_{K} \rightarrow 0$ the flow closely resembles that over an impermeable boundary, while for increasing $R e_{K}$ flow characteristics of the highly permeable boundary become increasingly apparent. More importantly, the conceptual linking between the impermeable boundary and the highly permeable boundary allows for a universal description (based on $R e_{K}$ ) of flows over granular beds.

These results have implications on the current use of popular interfacial transport models. The DBL model, for instance, assumes sediments to be impenetrable by the flow and cannot describe such features as a non-zero Brinkman layer thickness and a non-zero interfacial velocity. Increasing $R e_{K}$ even leads to the penetration of turbulent fluctuations and turbulent shear stress into the sediment, which clearly conflicts with the concept of a diffusive boundary layer above the SWI. While slip models do allow flow penetration, they rely heavily on experimental verification as the models require input of the interfacial velocity, the Brinkman layer thickness and/or the eddy viscosity profile. It has long been argued that $\delta_{b} \propto \sqrt{K}$, but this simple scaling is not fully supported by our results. Our results suggest that the performance of the slip model is highly dependent on the closure model used. Even if the Brinkman layer thickness can be reasonably predicted, the slip model needs further input on the contribution of turbulence across the interface, possibly in the form of an eddy viscosity model, to provide accuracy over a wide range of $R e_{K}$.

A novel modelling approach based on the $R e_{K}$ framework seems a strong alternative given the encouraging correlations between $R e_{K}$ and key interfacial transport properties. The continuous variation of these key properties from $R e_{K} \ll 1$ to $R e_{K} \gg 1$, suggests that such a model is potentially much broader in its practical application 
than the DBL and slip models, while being possibly more simple considering the dependency on a single parameter.

Though the results presented consider momentum transport only, the presence of a turbulent flow field across the interface has implications for the transport of mass. Our results show that turbulent activity, albeit small, is still present at the interface for the lowest value of $\operatorname{Re}_{K}\left(R e_{K}=0.36\right)$, and this can be significant for scalar transport, with tracer molecular diffusivities of $O\left(10^{-9} \mathrm{~m}^{2} \mathrm{~s}^{-1}\right)$. This is consistent with the data shown in figure 1, where only at $R e_{K} \sim O(0.01)$ is the eddy diffusivity equal to the molecular diffusivity. It suggests that for $R e_{K}>0.1$ turbulent activity is responsible for the enhanced diffusivities shown in figure 1 .

Further research is required on the impact of other sediment bed properties on the interfacial hydrodynamics. In particular, consideration of the length scale of the roughness elements, $k_{s}$, will allow extension of this framework beyond monodisperse granular beds, where $k_{s}$ is strongly correlated to $\sqrt{K}$. Comparison of the interfacial flow statistics observed here with those of a polydisperse or non-granular sediment bed would therefore be valuable, as the roughness scale could be varied while maintaining a constant permeability. Although this roughness scale is typically small compared to the boundary layer scale, differences in flow properties across this distance can be significant (contrast the left- and right-hand side of figure 13). Nevertheless, even across the roughness, the local flow properties are strongly dependent on the value of $R e_{K}$.

\section{Conclusions}

A basic framework has been proposed describing the hydrodynamics across the sediment-water interface in terms of the permeability Reynolds number, $R e_{K}$. This framework represents a combination of canonical flow typologies, namely impermeable boundary layer flow $\left(R e_{K} \ll 1\right)$ and highly permeable canopy flows $\left(R e_{K} \gg 1\right)$. As both flow typologies are characterised by distinct features, the framework suggests that flow at the sediment-water interface in aquatic systems (typically at $\operatorname{Re}_{K} \sim O(0.001-10)$ ) represents a mixture of the flow characteristics in either limit.

This framework is supported by novel experimental results across the SWI, highlighted by the existence of flow and shear stress penetration into the sediment bed and the presence of a velocity inflection point above the SWI. These features and other statistics at the SWI are consistent with the variation of the wall-blocking effect and show a strong correlation with $R e_{K}$. Many of these properties become independent of $R e_{K}$ as it increases, tending towards behaviour found in flows over highly permeable boundaries (i.e. canopies). These results suggest that a transition in interfacial flow properties occurs at $R e_{K} \simeq 1-2$, beyond which the turbulent shear stress starts to dominate the total shear stress at the interface, the turbulent penetration into the sediment becomes comparable to that of the mean flow and the inflectional velocity and turbulence intensities show good agreement with the predictive relations suggested for highly permeable boundaries.

The explicit findings of turbulence penetration into the sediment bed has major consequences for the application of mass and momentum transport models. The most common, the DBL model, does not account for the penetration of flow across the interface, let alone turbulence, making its application limited to $\operatorname{Re}_{K} \sim O(0.01)$. Moreover, models that do account for flow penetration, such as the slip model, are highly sensitive to the definition of the interface and employ boundary conditions that 
are found to be erroneous here. One such example is the Brinkman layer thickness, whose ratio to $\sqrt{K}$ is often taken to be constant. Here, however, it is shown to exhibit a strong correlation with $\operatorname{Re}_{K}$. The results presented here suggest that future predictive models for sediment-bounded flow should be based on this $R_{K}$ framework, which could condense the wide range of currently employed models that require complex input and calibration.

\section{Acknowledgements}

The authors would like to thank the anonymous reviewers whose insightful comments have helped to improve this manuscript. This research was supported under the Australian Research Council's Discovery Projects funding scheme (project no. DP120102500).

\section{REFERENCES}

Adrian, R. J., Meinhart, C. D. \& Tomkins, C. D. 2000 Vortex organization in the outer region of the turbulent boundary layer. J. Fluid Mech. 422, 1-54.

Bai, K., Katz, J. \& Meneveau, C. 2015 Turbulent flow structure inside a canopy with complex multi-scale elements. Boundary-Layer Meteorol. 155 (3), 435-457.

Battin, T. J., Besemer, K., Bengtsson, M. M., Romani, A. M. \& Packmann, A. I. 2016 The ecology and biogeochemistry of stream biofilms. Nat. Rev. Microbiol. 14 (4), 251-263.

Bear, J. 1972 Dynamics of Fluids in Porous Media. Courier Dover.

Beavers, G. S. \& Joseph, D. D. 1967 Boundary conditions at a naturally permeable wall. J. Fluid Mech. 30 (01), 197-207.

Blasco, J., Saenz, V. \& Gómez-Parra, A. 2000 Heavy metal fluxes at the sediment-water interface of three coastal ecosystems from south-west of the iberian peninsula. Sci. Total Environ. 247 (2), 189-199.

Boudreau, B. P. 2001 Solute transport above the sediment-water interface. In The Benthic Boundary Layer: Transport Processes and Biogeochemistry, pp. 104-126. Oxford University Press.

Boulton, A. J., Findlay, S., Marmonier, P., Stanley, E. H. \& Valett, H. M. 1998 The functional significance of the hyporheic zone in streams and rivers. Annu. Rev. Ecol. Syst. 29, 59-81.

Breugem, W. P., Boersma, B. J. \& Uittenbogatrd, R. E. 2006 The influence of wall permeability on turbulent channel flow. J. Fluid Mech. 562 (1), 35-72.

Chandler, I. D., Guymer, I., Pearson, J. M. \& VAn Egmond, R. 2016 Vertical variation of mixing within porous sediment beds below turbulent flows. Water Resour. Res. 52 (5), 3493-3509.

Ciceri, G., Maran, C., Martinotti, W. \& Queirazza, G. 1992 Geochemical cycling of heavy metals in a marine coastal area: benthic flux determination from pore water profiles and in situ measurements using benthic chambers. Hydrobiologia 235 (1), 501-517.

Cowen, E. A. \& Monismith, S. G. 1997 A hybrid digital particle tracking velocimetry technique. Exp. Fluids 22 (3), 199-211.

Detert, M., Klar, M., Wenka, T. \& Jirka, G. H. 2007 Pressure- and velocity-measurements above and within a porous gravel bed at the threshold of stability. Dev. Earth Surf. Processes 11, 85-105.

DiAZ, R. J. 2001 Overview of hypoxia around the world. J. Environ. Qual. 30 (2), 275-281.

GhisAlberti, M. 2009 Obstructed shear flows: similarities across systems and scales. J. Fluid Mech. 641, 51-61.

Ghisalberti, M. \& NePf, H. M. 2002 Mixing layers and coherent structures in vegetated aquatic flows. J. Geophys. Res. 107 (C2), 3-1-3-11.

Goharzadeh, A., Khalili, A. \& Jørgensen, B. B. 2005 Transition layer thickness at a fluidporous interface. Phys. Fluids 17 (5), 057102. 
Goyeau, B., Lhuillier, D., Gobin, D. \& Velarde, M. G. 2003 Momentum transport at a fluid-porous interface. Intl J. Heat Mass Transfer 46 (21), 4071-4081.

Grant, S. B., Stewardson, M. J. \& Marusic, I. 2012 Effective diffusivity and mass flux across the sediment-water interface in streams. Water Resour. Res. 48 (5), W05548.

GÜsS, S. 1998 Oxygen uptake at the sediment-water interface simultaneously measured using a flux chamber method and microelectrodes: must a diffusive boundary layer exist? Estuar. Coast Shelf Sci. 46 (1), 143-156.

Häfeli, R., Altheimer, M., Butscher, D. \& Von Rohr, P. R. 2014 PIV study of flow through porous structure using refractive index matching. Exp. Fluids 55 (5), 1-13.

Ho, C. M. \& Huerre, P. 1984 Perturbed free shear layers. Annu. Rev. Fluid Mech. 16 (1), 365-422.

Hondzo, M., Feyaerts, T., Donovan, R.\& O'Connor, B. L. 2005 Universal scaling of dissolved oxygen distribution at the sediment-water interface: a power law. Limnol. Oceanogr. 50 (5), $1667-1676$.

Horritt, M. S. \& BAtes, P. D. 2002 Evaluation of 1D and 2D numerical models for predicting river flood inundation. J. Hydrol. 268 (1), 87-99.

Jiménez, J. 2004 Turbulent flows over rough walls. Annu. Rev. Fluid Mech. 36, 173-196.

Jorgensen, B. B. \& Des Marais, D. J. 1990 The diffusive boundary layer of sediments: oxygen microgradients over a microbial mat. Limnol. Oceanogr. 35, 1343-1355.

Kim, J., Moin, P. \& Moser, R. 1987 Turbulence statistics in fully developed channel flow at low Reynolds number. J. Fluid Mech. 177, 133-166.

Lorke, A., Muller, B., Maerki, M. \& Wuest, A. 2003 Breathing sediments: The control of diffusive transport across the sediment-water interface by periodic boundary-layer turbulence. Limnol. Oceanogr. 48 (6), 2077-2085.

Manes, C., Poggi, D. \& Ridolfi, L. $2011 a$ Turbulent boundary layers over permeable walls: scaling and near-wall structure. J. Fluid Mech. 687, 141-170.

Manes, C., Pokrajac, D., Nikora, V. I., Ridolfi, L. \& Poggi, D. $2011 b$ Turbulent friction in flows over permeable walls. Geophys. Res. Lett. 38 (3), L03402.

Manes, C., Ridolfi, L. \& Katul, G. 2012 A phenomenological model to describe turbulent friction in permeable-wall flows. Geophys. Res. Lett. 39 (14), L14403.

Marusic, I., Mathis, R. \& Hutchins, N. 2010 Predictive model for wall-bounded turbulent flow. Science 329 (5988), 193-196.

Mathis, R., Marusic, I., Cabrit, O., Jones, N. L. \& IVey, G. N. 2014 Modelling bed shear-stress fluctuations in a shallow tidal river. J. Geophys. Res.-Oceans 119, 3185-3199.

Mignot, E., Barthelemy, E. \& Hurther, D. 2009 Double-averaging analysis and local flow characterization of near-bed turbulence in gravel-bed channel flows. J. Fluid Mech. 618, 279-303.

Nelson, J. M., Shreve, R. L., Mclean, S. R. \& Drake, T. G. 1995 Role of near-bed turbulence structure in bed load transport and bed form mechanics. Water Resour. Res. 31 (8), 2071-2086.

NePf, H. M. 2012 Flow and transport in regions with aquatic vegetation. Annu. Rev. Fluid Mech. 44, 123-142.

Nezu, I. \& NaKagawa, H. 1993 Turbulence in Open-Channel Flows. A.A. Balkema.

Nikora, V., Goring, D., McEwAn, I. \& Griffiths, G. 2001 Spatially averaged open-channel flow over rough bed. J. Hydraul. Engng 127 (2), 123-133.

Nikora, V., Koll, K., McEwan, I., McLean, S. \& DitTrich, A. 2004 Velocity distribution in the roughness layer of rough-bed flows. J. Hydraul. Engng 130 (10), 1036-1042.

Nikora, V., McEwan, I., Mclean, S., Coleman, S., Pokrajac, D. \& Walters, R. 2007 Doubleaveraging concept for rough-bed open-channel and overland flows: theoretical background. J. Hydraul. Engng 133 (8), 873-883.

Nokes, R. 2016 Streams. System Theory and Design. University of Canterbury, version 2.05.

O'Connor, B. L. \& HARVEY, J. W. 2008 Scaling hyporheic exchange and its influence on biogeochemical reactions in aquatic ecosystems. Water Resour. Res. 44 (12), W12423.

Packman, A. I., Salehin, M. \& Zaramella, M. 2004 Hyporheic exchange with gravel beds: basic hydrodynamic interactions and bedform-induced advective flows. J. Hydraul. Engng 130 (7), 647-656. 
Perot, B. \& Moin, P. 1995 Shear-free turbulent boundary layers. Part 1. Physical insights into near-wall turbulence. J. Fluid Mech. 295, 199-227.

Poggi, D., Porporato, A., Ridolfi, L., Albertson, J. D. \& Katul, G. G. 2004 The effect of vegetation density on canopy sub-layer turbulence. Boundary-Layer Meteorol. 111 (3), 565-587.

Pokrajac, D, Finnigan, J. J., Manes, C., McEwan, I. K. \& Nikora, V. I. 2006 On the definition of the shear velocity in rough bed open channel flows. In Proc. Intl. Conference on Flucial Hydraulics River Flow 2006, Lisbon, vol. 1, pp. 89-98. CRC Press.

Prinos, P., Sofialidis, D. \& Keramaris, E. 2003 Turbulent flow over and within a porous bed. J. Hydraul. Engng 129 (9), 720-733.

Rabalais, N. N., Smith, L. E., Harper, D. E. \& Justic, D. 2001 Effects of seasonal hypoxia on continental shelf benthos. In Coastal Hypoxia: Consequences for Living Resources and Ecosystems (ed. N. N. Rabalais \& R. E. Turner), American Geophysical Union.

Raupach, M. R., Finnigan, J. J. \& BRUneI, Y. 1996 Coherent eddies and turbulence in vegetation canopies: the mixing-layer analogy. Boundary-Layer Meteorol. 78 (3-4), 351-382.

Rosgen, D. L. 1994 A classification of natural rivers. Catena 22 (3), 169-199.

Roy, H., Huettel, M. \& Jorgensen, B. B. 2004 Transmission of oxygen concentration fluctuations through the diffusive boundary layer overlying aquatic sediments. Limnol. Oceanogr. 49 (3), 686-692.

Ruff, J. F. \& Gelhar, L. W. 1972 Turbulent shear flow in porous boundary. J. Engng Mech. 98, 975-991.

SAfFMAN, P. G. 1971 On the boundary condition at the surface of a porous medium. Stud. Appl. Maths 50 (2), 93-101.

SChlichting, H. 1979 Boundary-Layer Theory. McGraw-Hill.

Smalley, R., Leonardi, S., Antonia, R., Djenidi, L. \& Orlandi, P. 2002 Reynolds stress anisotropy of turbulent rough wall layers. Exp. Fluids 33 (1), 31-37.

Suga, K., Matsumura, Y., Ashitaka, Y., Tominaga, S. \& Kaneda, M. 2010 Effects of wall permeability on turbulence. Intl J. Heat Fluid Flow 31 (6), 974-984.

Suga, K., Mori, M. \& KANEDA, M. 2011 Vortex structure of turbulence over permeable walls. Intl J. Heat Fluid Flow 32 (3), 586-595.

Tennekes, H. \& Lumley, J. L. 1972 A First Course in Turbulence. MIT Press.

Tilton, N. \& Cortelezzi, L. 2008 Linear stability analysis of pressure-driven flows in channels with porous walls. J. Fluid Mech. 604, 411-445.

Uzol, O., Chow, Y. C., Katz, J. \& Meneveau, C. 2002 Unobstructed particle image velocimetry measurements within an axial turbo-pump using liquid and blades with matched refractive indices. Exp. Fluids 33 (6), 909-919.

White, B. L. \& NePF, H. M. 2007 Shear instability and coherent structures in shallow flow adjacent to a porous layer. J. Fluid Mech. 593, 1-32.

Wilson, A. M., Huettel, M. \& Klein, S. 2008 Grain size and depositional environment as predictors of permeability in coastal marine sands. Estuar. Coast Shelf Sci. 80 (1), 193-199. 